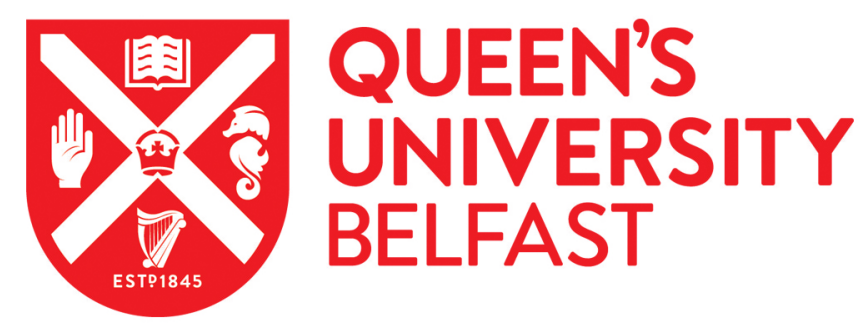

\title{
Multi-Beam Multiplexing Design for Arbitrary Directions Based on the Interleaved Subarray Architecture
}

Zhang, J., Liu, W., Gu, C., Gao, S. S., \& Luo, Q. (2020). Multi-Beam Multiplexing Design for Arbitrary Directions Based on the Interleaved Subarray Architecture. IEEE Transactions on Vehicular Technology, 69(10), 1122011232. [9138782]. https://doi.org/10.1109/tvt.2020.3008535

Published in:

IEEE Transactions on Vehicular Technology

Document Version:

Peer reviewed version

Queen's University Belfast - Research Portal:

Link to publication record in Queen's University Belfast Research Portal

Publisher rights

Copyright 2020 IEEE. This work is made available online in accordance with the publisher's policies. Please refer to any applicable terms of use of the publisher.

\section{General rights}

Copyright for the publications made accessible via the Queen's University Belfast Research Portal is retained by the author(s) and / or other copyright owners and it is a condition of accessing these publications that users recognise and abide by the legal requirements associated with these rights.

Take down policy

The Research Portal is Queen's institutional repository that provides access to Queen's research output. Every effort has been made to ensure that content in the Research Portal does not infringe any person's rights, or applicable UK laws. If you discover content in the Research Portal that you believe breaches copyright or violates any law, please contact openaccess@qub.ac.uk. 


\title{
Multi-Beam Multiplexing Design for Arbitrary Directions Based on the Interleaved Subarray Architecture
}

\author{
Junwei Zhang, Student Member, IEEE, Wei Liu, Senior Member, IEEE, Chao Gu, Steven Gao, Fellow, IEEE, and \\ Qi Luo, Senior Member, IEEE
}

\begin{abstract}
Through a common set of analogue coefficients and a simple digital coding scheme in the form of ones and minus ones, a previous technique can only multiplex two beams whose directions satisfy a fixed relationship. In this work, two novel designs are proposed, which together with the corresponding inter-subarray coding schemes, can achieve multi-beam multiplexing for arbitrary directions to serve corresponding users. In the first design, based on the relationship of directions between the two required beams, the adjacent antenna spacing is regarded as a variable to be determined, while in the second design, the adjacent antenna spacing is fixed and an alternate optimisation procedure is proposed to solve the problem based on a leastsquare formulation. Designed examples based on uniform linear arrays and uniform planar arrays are provided to demonstrate the effectiveness of the proposed methods.
\end{abstract}

Index Terms-interleaved subarray architecture, intersubarray coding, beam multiplexing, adjacent antenna spacing, arbitrary directions

\section{INTRODUCTION}

$\mathbf{T}$ WO key enabling technologies of the next-generation (5-G) communication systems are massive MIMO and mmWave communication [1] and both require the employment of numerous antennas working at high frequencies with a wide bandwidth. If the traditional beamforming process is implemented completely in the digital domain, the extremely high cost associated with the large number of high-speed analogue to digital converters (ADCs) and the high-level power consumption will cause it practically infeasible.

One solution to the problem is to employ the well-known hybrid beamforming structure [2]-[12], which is a combination of the analogue beamforming technique [13]-[15] and the digital beamforming technique [16]. Analogue beamforming is employed first to reduce the number of analogue channels, which are then converted into the digital domain via a reduced number of ADCs, and after that digital beamforming can then be employed.

Various hybrid beamforming structures have been proposed in the past and one of them is the sub-aperture based hybrid beamformer [10], [12], [17]-[20]. There are mainly two types

Copyright (c) 2020 IEEE. Personal use of this material is permitted. However, permission to use this material for any other purposes must be obtained from the IEEE by sending a request to pubs-permissions@ieee.org

J. W. Zhang and W. Liu are with the Department of Electronic and Electrical Engineering, University of Sheffield, Sheffield, S1 3JD, UK(e-mail jzhang96@sheffield.ac.uk, w.liu@ sheffield.ac.uk)

C. Gu is with the ECIT Institute, Queen's University Belfast, Belfast, BT3 9DT, UK(e-mail: chao.gu@qub.ac.uk)

S. Gao and Q. Luo are with the School of Engineering and Digital Arts, University of Kent, Canterbury, CT2 7NZ, UK(e-mail: s.gao@kent.ac.uk, q,luo@kent.ac.uk) of implementation for the subarray scheme: one is the sideby-side type or localised architecture and the other one is the interleaved architecture [21], [22]. In the side-by-side structure, all the antennas belonging to the same subarray are located within a local region next to each other; as a result, the beam width generated by this architecture is comparatively wide. For the interleaved architecture, the antennas of each subarray are distributed over a much larger aperture and the spacing between adjacent subarray antennas is much larger than the standard array spacing. Thus, a much narrower beam can be formed by the interleaved structure, which makes it a good candidate for beam multiplexing; however, this narrow beamwidth is achieved at the cost of generating high sidelobes or even grating lobes or spatial aliasing, although this effect can be suppressed at a later stage by digital beamforming technique to some extent with improved desired beam gain [23]-[25].

Recently, an interesting hybrid beamforming approach which involves multiplexing two beams was proposed in [26]. However, as pointed out later in this paper, a limitation of the proposed method is that the directions of the two beams must satisfy a specific relationship and therefore it is not suitable for users located at arbitrary directions. In this work, we propose two effective designs to achieve multiple-beam multiplexing for arbitrary directions. For the first design, the adjacent antenna spacing is treated as a variable which is designed according to the specific relationship between the required two beams, but a clear issue is that it may not be practical to constantly change the spacing to meet the needs of changing user directions, although this could be solved by performing antenna switching through preparing more antennas at predefined candidate locations than necessary for a required beam width [27]. In the second design, to deal with the issue in the first design, the antenna spacing is fixed and independent of beam directions, and we optimise the beamformer coefficients in multiple subarrays for best approximation between the designed and desired beam responses in a least-squares formulation. Compared to our earlier conference publication [27], in addition to extending the work from two beams to multiple beams and proposing a general design approach, i.e., the second design, we have also considered the design based on uniform planar arrays (UPAs), which will be widely used in mmWave communications. As demonstrated by design examples, the two designs can generate multiple high-quality beams with arbitrary directions to serve corresponding users.

The remaining part of this paper is organised as follows. A review of the interleaved subarray architecture is presented in Section II. An inter-subarray coding scheme with varying 


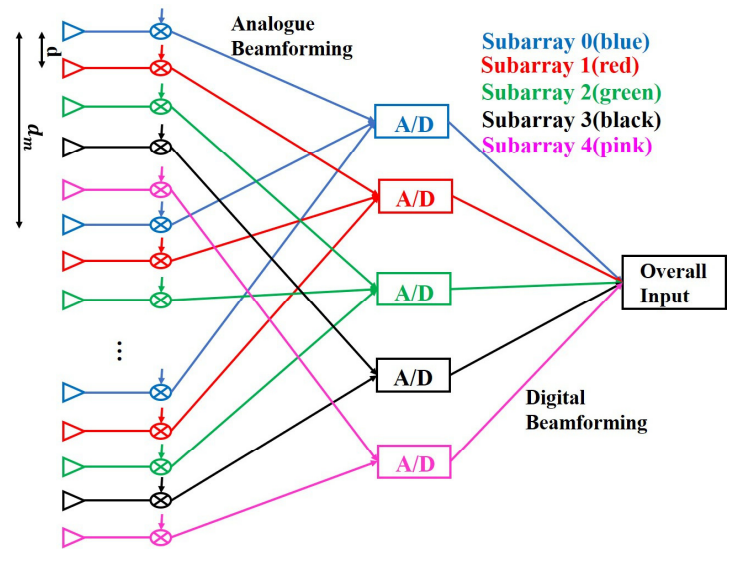

Fig. 1. An interleaved ULA subarray based hybrid beamforming structure.

antenna spacing for two users in arbitrary directions is described in Section III and the design based on a fixed antenna spacing for multiple users is considered in Section IV. Design examples are provided in Section $\mathrm{V}$ and conclusions are drawn in Section VI.

\section{THE INTERLEAVED SUBARRAY ARCHITECTURE BASED ON LINEAR ARRAYS}

The interleaved subarray structure based on an $N$-element uniform linear array (ULA) is shown in Fig. 1, where the adjacent antenna spacing is $d$. Suppose the $N$ elements of the ULA are divided into $M$ interleaved subarrays. Then, each subarray consists of $N_{s}=N / M$ antennas with an adjacent antenna spacing $d_{m}=M d$. The phase shift between adjacent subarrays is $e^{j 2 \pi \frac{d}{\lambda} \sin \theta}$, where the direction of angle $\theta$ is measured from the broadside of the array.

The beam response $P_{m}\left(\theta, \varphi_{m}\right), m=0,1, \cdots, M-1$, generated by the $m$-th subarray pointing to the direction $\varphi_{m}$ is given by

$$
\begin{gathered}
P_{m}\left(\theta, \varphi_{m}\right)=e^{j 2 \pi p_{m} \sin \theta} \\
\sum_{n=0}^{N_{s}-1} w_{m, n}\left(\varphi_{m}\right) \exp \left[j 2 \pi \frac{d_{m}}{\lambda} n \sin \theta\right],
\end{gathered}
$$

where $p_{m}=m \frac{d}{\lambda}$ denotes the initial location of the $m$-th subarray in terms of the signal wavelength $\lambda$, and $w_{m, n}\left(\varphi_{m}\right)$ denotes the analogue weighting factor of the $n$-th antenna of the $m$-th subarray for the main beam direction pointing to $\varphi_{m}$. Through inter-subarray coding, the $M$ beams are generated by the $M$-subarray based hybrid beamforming scheme configured by the interleaved subarray architecture. The overall beam response using the $M$ subarrays with a main beam in the direction $\varphi_{x}$ is

$$
\begin{gathered}
P\left(\theta, \varphi_{x}\right)=\sum_{m=0}^{M-1} w_{D, x, m} P_{m}\left(\theta, \varphi_{m}\right)=\sum_{m=0}^{M-1} w_{D, x, m} \\
e^{j 2 \pi p_{m} \sin \theta} \sum_{n=0}^{N_{s}-1} w_{m, n}\left(\varphi_{m}\right) \exp \left[j 2 \pi \frac{d_{m}}{\lambda} n \sin \theta\right],
\end{gathered}
$$

where $w_{D, x, m}$ is the digital weighting factor for the $m$-th subarray.

\section{THE PROPOSED DESIGN WITH VARYING ANTENNA SPACING AND ASSOCIATED INTER-SUBARRAY CODING SCHEME}

In [26], the two-user scenario is considered, where there are two subarrays in total $(M=2)$ and two beams are generated by an inter-subarray coding scheme with a linear combination of analogue weighting factors for two subarrays. With $d=\frac{\lambda}{2}$, the analogue weighting factors of the zeroth and first subarrays are given by

$$
\begin{aligned}
& w_{0, n}\left(\varphi_{0}\right)=e^{-j 2 \pi n \sin \varphi_{0}}, \\
& w_{1, n}\left(\varphi_{1}\right)=e^{-j 2 \pi\left(n+\frac{1}{2}\right) \sin \varphi_{1}},
\end{aligned}
$$

where $\varphi_{0}$ and $\varphi_{1}$ are the desired directions for two users. This interleaved-subarray beamforming system generates the zeroth user's own beam naturally and the first user's beam in the direction of the zeroth user's grating lobe and vice versa, and as a result, data for the zeroth user is divided in opposite phase and data for the first user is divided in the same phase. Specifically, as proposed in [23], [26], the digital beamformer coefficient vector $\mathbf{w}_{\mathrm{D}, \mathrm{x}}(x=0,1)$ can be characterized as follows

$$
\begin{aligned}
& \mathbf{w}_{\mathrm{D}, 0}=\left[w_{\mathrm{D}, 0,0}, w_{\mathrm{D}, 0,1}\right]=\left[\begin{array}{rr}
1 & -1
\end{array}\right], \\
& \mathbf{w}_{\mathrm{D}, 1}=\left[w_{\mathrm{D}, 1,0}, w_{\mathrm{D}, 1,1}\right]=\left[\begin{array}{ll}
1 & 1
\end{array}\right] .
\end{aligned}
$$

Finally, the responses of two generated beams are

$$
\begin{aligned}
P\left(\theta, \varphi_{0}\right)= & \sum_{n=0}^{N_{s}-1} e^{j 2 \pi n\left(\sin \theta-\sin \varphi_{0}\right)}- \\
& \sum_{n=0}^{N_{s}-1} e^{j 2 \pi\left(n+\frac{1}{2}\right)\left(\sin \theta-\sin \varphi_{1}\right)}, \\
P\left(\theta, \varphi_{1}\right)= & \sum_{n=0}^{N_{s}-1} e^{j 2 \pi n\left(\sin \theta-\sin \varphi_{0}\right)}+ \\
& \sum_{n=0}^{N_{s}-1} e^{j 2 \pi\left(n+\frac{1}{2}\right)\left(\sin \theta-\sin \varphi_{1}\right)} .
\end{aligned}
$$

Ideally (6) should form a beam pointing to direction $\varphi_{0}$ while (7) forms a beam pointing to direction $\varphi_{1}$. Generally, for a ULA of $2 N_{s}$ antennas with adjacent antenna spacing $d=\frac{\lambda}{2}$, to have a beam response with its main beam direction in $\varphi_{x}$, one way is to steer the broadside main beam with uniform weighting as follows,

$$
P\left(\theta, \varphi_{x}\right)=\sum_{n=0}^{2 N_{s}-1} e^{j \pi n\left(\sin \theta-\sin \varphi_{x}\right)} .
$$

The key is to find some appropriate parameters so that (6) and (7) will be transformed into the form of (8) with $x=0,1$, respectively. To realise this, (6) is expanded into the following form

$$
\begin{gathered}
P\left(\theta, \varphi_{0}\right)=\sum_{n=0}^{N_{s}-1} e^{j 2 \pi n\left(\sin \theta-\sin \varphi_{0}\right)}- \\
\sum_{n=0}^{N_{s}-1} e^{j \pi(2 n+1)\left(\sin \theta-\sin \varphi_{0}\right)} e^{j \pi(2 n+1)\left(\sin \varphi_{0}-\sin \varphi_{1}\right)} .
\end{gathered}
$$


It can be observed that as long as

$$
e^{j \pi\left(\sin \varphi_{0}-\sin \varphi_{1}\right)}=-1
$$

or equivalently

$$
\left|\sin \varphi_{0}-\sin \varphi_{1}\right|=1 \text {, }
$$

(6) and (7) will be converted into the form of (8) with $x=$ 0,1 , respectively. Hence, in [26], [28], there is an important limitation to this scheme: the two user directions cannot be arbitrary and have to follow this specific relationship. Although in practice, it is hard to find two user directions meeting exactly this relationship, it theoretically verifies for the first time that it is possible to use a simple hybrid analogue and digital beamforming technique to produce two beams without spatial aliasing.

Now in the following we try to overcome the restriction of the scheme in [26], [28] and design a new scheme with two interleaved subarrays which can form beams in two arbitrary directions. First, given the simple form of the digital beamformer coefficients $\mathbf{w}_{\mathrm{D}, 0}$ and $\mathbf{w}_{\mathrm{D}, 1}$ in (4) and (5), respectively, they are still adopted in the new scheme. Consider a general ULA with adjacent spacing $d=\alpha \frac{\lambda}{2}$, where $\alpha$ is a coefficient whose value will be determined later. Hence, the adjacent antenna spacing for the $m$-th subarray is supposed as

$$
d_{m}=M d=M \alpha \frac{\lambda}{2}=\alpha \lambda,
$$

where $M=2$ has been used for two subarrays and the initial position $p_{m}$ of the $m$-th subarray is

$$
p_{m}=m \frac{d}{\lambda}=m \frac{\alpha}{2} \text {. }
$$

To form a beam to direction $\varphi_{x}$ using a ULA of $2 N_{s}$ antennas with adjacent antenna spacing $d=\alpha \frac{\lambda}{2}$, similar to (8), the desired beam response for the $x$-th beam can be achieved by beam steering in combination with uniform weighting as follows

$$
P\left(\theta, \varphi_{x}\right)=\sum_{n=0}^{2 N_{s}-1} e^{j \alpha \pi n\left(\sin \theta-\sin \varphi_{x}\right)} .
$$

Moreover, based on (2), the new antenna analogue weighting factors for the zeroth and first subarrays can be chosen to compensate for the phase difference corresponding to look directions only with uniform magnitudes as follows

$$
\begin{aligned}
& w_{0, n}\left(\varphi_{0}\right)=e^{-j 2 \alpha \pi n \sin \varphi_{0}}, \\
& w_{1, n}\left(\varphi_{1}\right)=e^{-j 2 \alpha \pi\left(n+\frac{1}{2}\right) \sin \varphi_{1}} .
\end{aligned}
$$

Then, the designed beam responses for the two beams are given by

$$
\begin{aligned}
P\left(\theta, \varphi_{0}\right)= & \sum_{n=0}^{N_{s}-1} e^{j 2 \alpha \pi n\left(\sin \theta-\sin \varphi_{0}\right)}- \\
P\left(\theta, \varphi_{1}\right)= & \sum_{n=0}^{N_{s}-1} e^{j 2 \alpha \pi\left(n+\frac{1}{2}\right)\left(\sin \theta-\sin \varphi_{1}\right)}, \\
& \sum_{n=0}^{N_{s}-1} e^{j 2 \alpha \pi n\left(\sin \theta-\sin \varphi_{0}\right)}+
\end{aligned}
$$

To find $\alpha$, similar to (9), we modify (16) into

$$
\begin{gathered}
P\left(\theta, \varphi_{0}\right)=\sum_{n=0}^{N_{s}-1} e^{j 2 \alpha \pi n\left(\sin \theta-\sin \varphi_{0}\right)}- \\
\sum_{n=0}^{N_{s}-1} e^{j \alpha \pi(2 n+1)\left(\sin \theta-\sin \varphi_{0}\right)} e^{j \alpha \pi(2 n+1)\left(\sin \varphi_{0}-\sin \varphi_{1}\right)} .
\end{gathered}
$$

For (18) to match (14) when $x=0$, similar to (10), one solution can be obtained by satisfying

$$
e^{j \alpha \pi\left(\sin \varphi_{0}-\sin \varphi_{1}\right)}=-1 \text {. }
$$

For arbitrary $\varphi_{0}$ and $\varphi_{1}$, the value of $\alpha$ can then be calculated by

$$
\alpha=\frac{1}{\left|\sin \varphi_{0}-\sin \varphi_{1}\right|} .
$$

\section{THE PROPOSED DESIGN WITH FIXED ANTENNA SPACING AND ASSOCIATED INTER-SUBARRAY CODING SCHEME}

As mentioned, it may not be practical to constantly change the spacing to meet the needs of changing user directions. In this section, the beamformer coefficients will be redesigned, while maintaining the adjacent antenna spacing $d$ as a fixed value. Although the approach introduced in this section in general can be applied to arbitrary number of beams with arbitrary directions, it is difficult to have a single general formulation to cover all the cases. As a result, we will only consider the two-beam and three-beam cases as representative examples and following the same approach, it can be extended to more than three beams without difficulty. Moreover, as uniform planar arrays (UPAs) will be widely used for mmWave communications, we will also consider a design based on UPAs to show that the approach can also be applied to twodimensional arrays.

\section{A. Uniform Linear Array}

\section{i) Two beams}

The steering vector of the $m$-th interleaved subarray is given by

$$
\begin{aligned}
\mathbf{s}_{\mathrm{m}}(\theta)= & {\left[e^{j 2 \pi m \frac{d}{\lambda} \sin \theta}, e^{j 2 \pi \frac{(m+M) d}{\lambda} \sin \theta},\right.} \\
& \left.\ldots, e^{j 2 \pi\left(m+M\left(N_{s}-1\right)\right) \frac{d}{\lambda} \sin \theta}\right]^{\mathrm{T}}
\end{aligned}
$$

where $[.]^{\mathrm{T}}$ denotes the transpose operation with $m=\{0,1\}$. Then, the beam response generated by the $m$-th subarray is

$$
P_{\mathrm{m}}(\theta)=\mathbf{w}_{\mathrm{m}}^{\mathrm{H}} \mathbf{s}_{m}(\theta),
$$

where $[.]^{\mathrm{H}}$ denotes the Hermitian transpose and $\mathbf{w}_{\mathrm{m}}$ denotes the analogue coefficients for the $m$-th subarray containing corresponding coefficients

$$
\mathbf{w}_{\mathrm{m}}=\left[w_{\mathrm{m}, 0}, w_{\mathrm{m}, 1}, \ldots, w_{\mathrm{m}, \mathrm{N}_{\mathrm{s}}-1}\right]^{\mathrm{T}} .
$$

We employ a general digital coding scheme in the interleaved structure, whose coefficients for the beam in direction $\varphi_{x}$ is given by

$$
\mathbf{w}_{\mathrm{D}, \mathrm{x}}=\left[w_{\mathrm{D}, \mathrm{x}, 0}, w_{\mathrm{D}, \mathrm{x}, 1}\right]=\left[\begin{array}{ll}
a_{x, 0} & a_{x, 1}
\end{array}\right]
$$


where $a_{x, 0}, a_{x, 1}(x=\{0,1\})$ are four digital coefficients to be determined later. So the designed beam response for the beam pointing to direction $\varphi_{x}$ in vector form is

$$
\begin{aligned}
P_{\varphi_{\mathrm{x}}}(\theta) & =a_{x, 0} P_{0}(\theta)+a_{x, 1} P_{1}(\theta) \\
& =a_{x, 0} \mathbf{w}_{0}^{\mathrm{H}} \mathbf{s}_{0}(\theta)+a_{x, 1} \mathbf{w}_{1}^{\mathrm{H}} \mathbf{s}_{1}(\theta) .
\end{aligned}
$$

The following cost function based on least squares (LS) formulation is used in the design [16], [29], [30]

$$
J_{\mathrm{LS}}=\int_{\theta \in \Theta} F(\theta)|H(\theta)-D(\theta)|^{2} d \theta,
$$

where $\Theta$ denotes the overall angle range of interest, $F(\theta)$ is a positive real weighting function, and $H(\theta)$ and $D(\theta)$ are the designed and desired beam responses, respectively. In our designs, without loss of generality, we use $F(\theta)=1-\beta$ and $D(\theta)=1$ over the mainlobe region (one single direction) and $F(\theta)=\beta$ and $D(\theta)=0$ over the sidelobe region for each beam, where $\beta$ is the trade-off parameter between the mainlobe and sidelobe regions. To balance the minimisation in the mainlobe and sidelobe regions for two beams, $N_{g}$ is introduced to represent the number of sample points in the sidelobe region for each beam. A specific cost formulation combining the above two beams is given by

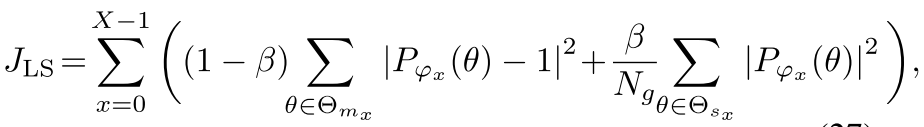

where $\Theta_{s_{x}}$ and $\Theta_{m_{x}}$ denote the sidelobe and mainlobe regions for the $x$-th beam and $X$ denotes the number of the designed beams with $X=2$ in this case. Substituting (25) into (27), the cost function $J_{\mathrm{LS}}$ can be rewritten as

$$
\begin{aligned}
J_{\mathrm{LS}}=\sum_{x=0}^{X-1} & \left((1-\beta) \sum_{\theta \in \Theta_{m_{x}}}\left|a_{x, 0} \mathbf{w}_{0}^{\mathrm{H}} \mathbf{s}_{0}(\theta)+a_{x, 1} \mathbf{w}_{1}^{\mathrm{H}} \mathbf{s}_{1}(\theta)-1\right|^{2}\right. \\
& \left.+\left(\beta / N_{g}\right) \sum_{\theta \in \Theta_{s_{x}}}\left|a_{x, 0} \mathbf{w}_{0}^{\mathrm{H}} \mathbf{s}_{0}(\theta)+a_{x, 1} \mathbf{w}_{1}^{\mathrm{H}} \mathbf{s}_{1}(\theta)\right|^{2}\right)
\end{aligned}
$$

which can be expanded as

$$
\begin{aligned}
& J_{\mathrm{LS}}=\sum_{x=0}^{X-1}\left(a_{x, 0}^{2} \mathbf{w}_{0}^{\mathrm{H}} \mathbf{Q}_{\mathrm{LS} \mathrm{x}_{0}} \mathbf{w}_{0}+a_{x, 1}^{2} \mathbf{w}_{1}^{\mathrm{H}} \mathbf{Q}_{\mathrm{LS} \mathrm{x}_{\mathbf{1}}} \mathbf{w}_{1}\right. \\
& +a_{x, 0} a_{x, 1}\left(\mathbf{w}_{0}^{\mathrm{H}} \mathbf{P}_{\mathrm{LS}_{\mathrm{x} 0}} \mathbf{w}_{1}+\mathbf{w}_{1}^{\mathrm{H}} \mathbf{P}_{\mathrm{LS}_{\mathrm{x} 1}} \mathbf{w}_{0}\right) \\
& -a_{x, 0}\left(\mathbf{w}_{0}^{\mathrm{H}} \mathbf{z}_{\mathrm{LS} \mathrm{x}_{\mathbf{x}}}+\mathbf{z}_{\mathrm{LS} \mathrm{x}_{\mathbf{x}}}^{\mathrm{H}} \mathbf{w}_{0}\right)-a_{x, 1}\left(\mathbf{w}_{1}^{\mathrm{H}} \mathbf{z}_{\mathrm{LS}}+\mathbf{z}_{\mathrm{LS} \mathbf{x} 1}^{\mathrm{H}} \mathbf{w}_{1}\right) \\
& \left.+d_{\mathrm{LS}_{\mathrm{x}}}\right)
\end{aligned}
$$

with

$$
\begin{gathered}
\mathbf{Q}_{\mathrm{LS}}=(1-\beta) \sum_{\theta \in \Theta_{m_{\mathbf{x}}}} \mathbf{S}_{\mathrm{q}}(\theta)+\frac{\beta}{N_{g}} \sum_{\theta \in \Theta_{s_{\mathbf{x}}}} \mathbf{S}_{\mathrm{q}}(\theta), \\
\mathbf{P}_{\mathrm{LS}_{\mathbf{x} 0}}=(1-\beta) \sum_{\theta \in \Theta_{m_{\mathbf{x}}}} \mathbf{s}_{0}(\theta) \mathbf{s}_{1}(\theta)^{\mathrm{H}}+\frac{\beta}{N_{g}} \sum_{\theta \in \Theta_{s_{\mathbf{x}}}} \mathbf{s}_{0}(\theta) \mathbf{s}_{1}(\theta)^{\mathrm{H}}, \\
\mathbf{P}_{\mathrm{LS}_{\mathbf{x} 1}}=(1-\beta) \sum_{\theta \in \Theta_{m_{\mathbf{x}}}} \mathbf{s}_{1}(\theta) \mathbf{s}_{0}(\theta)^{\mathrm{H}}+\frac{\beta}{N_{g}} \sum_{\theta \in \Theta_{s_{\mathbf{x}}}} \mathbf{s}_{1}(\theta) \mathbf{s}_{0}(\theta)^{\mathrm{H}},
\end{gathered}
$$

$$
\begin{gathered}
\mathbf{z}_{\mathrm{LS}_{\mathrm{xq}}}=(1-\beta) \sum_{\theta \in \Theta_{m_{\mathrm{x}}}} \mathbf{s}_{\mathrm{q}}(\theta), \\
d_{\mathrm{LS}_{\mathrm{x}}}=(1-\beta) \sum_{\theta \in \Theta_{m_{\mathrm{x}}}} 1, \\
\mathbf{S}_{\mathrm{q}}(\theta)=\mathbf{s}_{\mathrm{q}}(\theta) \mathbf{s}_{\mathrm{q}}(\theta)^{\mathrm{H}}
\end{gathered}
$$

where $\{x, q\}=\{0,1\}$. Combining the analogue coefficients $\mathbf{w}_{0}$ and $\mathbf{w}_{1}$ into one vector, given by

$$
\mathbf{w}_{\mathrm{A}}=\left[\begin{array}{l}
\mathbf{w}_{0} \\
\mathbf{w}_{1}
\end{array}\right]
$$

equation (29) can be rewritten as

$$
\begin{aligned}
J_{\mathrm{LS}} & =\mathbf{w}_{\mathrm{A}}^{\mathrm{H}}\left(\mathbf{Q}_{\mathrm{LS}}+\mathbf{P}_{\mathrm{LS}} \tilde{\mathbf{I}}_{\mathrm{LS}}\right) \mathbf{w}_{\mathrm{A}}-\mathbf{w}_{\mathrm{A}}^{\mathrm{H}} \mathbf{z}_{\mathrm{LS}}-\mathbf{z}_{\mathrm{LS}}^{\mathrm{H}} \mathbf{w}_{\mathrm{A}}+d_{\mathrm{LS} 0} \\
& +d_{\mathrm{LS} 1}
\end{aligned}
$$

with

$$
\begin{gathered}
\mathbf{Q}_{\mathrm{LS}}=\left[\begin{array}{cc}
\mathbf{G}_{\mathrm{LS}_{0}} & \mathbf{0} \\
\mathbf{0} & \mathbf{G}_{\mathrm{LS}_{1}}
\end{array}\right] \\
\mathbf{P}_{\mathrm{LS}}=\left[\begin{array}{cc}
\mathbf{H}_{\mathrm{LS}} & \mathbf{0} \\
\mathbf{0} & \mathbf{H}_{\mathrm{LS}}
\end{array}\right] \\
\tilde{\mathbf{I}}_{\mathrm{LS}}=\left[\begin{array}{ll}
\mathbf{0} & \mathbf{1} \\
\mathbf{1} & \mathbf{0}
\end{array}\right] \\
\mathbf{z}_{\mathrm{LS}}=\left[\begin{array}{l}
a_{0,0} \mathbf{z}_{\mathrm{LS}}+a_{1,0} \mathbf{z}_{\mathrm{LS}} \\
a_{0,1} \mathbf{z}_{\mathrm{LS}}+a_{1,1} \mathbf{z}_{\mathrm{LS}}
\end{array}\right] \\
\mathbf{G}_{\mathrm{LS}}=a_{0, \mathrm{q}}^{2} \mathbf{Q}_{\mathrm{LS}}+a_{1, \mathrm{q}}^{2} \mathbf{Q}_{\mathrm{LS}} \\
\mathbf{H}_{\mathrm{LS}_{\mathrm{q}}}=a_{0,0} a_{0,1} \mathbf{P}_{\mathrm{LS}_{0 \mathrm{q}}}+a_{1,0} a_{1,1} \mathbf{P}_{\mathrm{LS}_{1 \mathrm{q}}}
\end{gathered}
$$

where $\mathbf{0}$ and $\mathbf{1}$ are $N_{s} \times N_{s}$ null and identity matrices, respectively. For a given set of $a_{\mathrm{x}, \mathrm{q}}(\{x, q\} \in\{0,1\})$, $\mathbf{w}_{\mathrm{A}}$ can be obtained by taking the gradient of the cost function in (37) with respect to $w_{A}$ and then setting it to zero, given by

$$
\mathbf{w}_{\mathrm{A}}=\left(\mathbf{Q}_{\mathrm{LS}}+\mathbf{P}_{\mathrm{LS}} \tilde{\mathbf{I}}_{\mathrm{LS}}\right)^{-1} \mathbf{z}_{\mathrm{LS}}
$$

Joint optimisation of the digital coefficients $a_{0,0}, a_{0,1}, a_{1,0}$, $a_{1,1}$ and the corresponding analogue coefficients $\mathbf{w}_{\mathrm{A}}$ can be achieved by the following iterative process:

1) First, via initialising the digital coefficients $a_{0,0}=1$, $a_{0,1}=-1, a_{1,0}=1$, and $a_{1,1}=1$, as in [26], the values of $\mathbf{w}_{\mathrm{A}}$ are obtained by substituting $a_{0,0}, a_{0,1}$, $a_{1,0}$, and $a_{1,1}$ into (44).

2) Given the obtained values of $w_{A}$ in step 1), we find the closed-form solution of digital coefficients $a_{0,0}, a_{0,1}$, $a_{1,0}, a_{1,1}$ by minimising the cost function (29), given by

$$
\begin{aligned}
& {\left[\begin{array}{l}
a_{\mathrm{x}, 0} \\
a_{\mathrm{x}, 1}
\end{array}\right]=\left[\begin{array}{cc}
\mathbf{w}_{\mathrm{A}}^{\mathrm{H}} \hat{\mathbf{Q}}_{\mathrm{LS} \mathrm{S}_{\mathrm{x} 0}} \mathbf{w}_{\mathrm{A}} & \mathbf{w}_{\mathrm{A}}^{\mathrm{H}} \hat{\mathbf{P}}_{\mathrm{LS}_{\mathbf{x}}} \tilde{\mathbf{I}}_{\mathrm{LS}} \mathbf{w}_{\mathrm{A}} \\
\mathbf{w}_{\mathrm{A}}^{\mathrm{H}} \hat{\mathbf{P}}_{\mathrm{LS}} \tilde{\mathbf{I}}_{\mathrm{LS}} \mathbf{w}_{\mathrm{A}} & \mathbf{w}_{\mathrm{A}}^{\mathrm{H}} \hat{\mathbf{Q}}_{\mathrm{LS}} \mathbf{w}_{\mathrm{A}}
\end{array}\right]^{-1}} \\
& {\left[\begin{array}{l}
\mathbf{w}_{\mathrm{A}}^{\mathrm{H}} \hat{\mathbf{z}}_{\mathrm{LS} \mathrm{x}_{0}}+\hat{\mathbf{z}}_{\mathrm{LS} \mathrm{S}_{\mathrm{x}}}^{\mathrm{H}} \mathbf{w}_{\mathrm{A}} \\
\mathbf{w}_{\mathrm{A}}^{\mathrm{H}} \hat{\mathbf{z}}_{\mathrm{LS} \mathrm{x} 1}+\hat{\mathbf{z}}_{\mathrm{LS} \mathrm{S}_{\mathrm{x}}}^{\mathrm{H}} \mathbf{w}_{\mathrm{A}}
\end{array}\right]}
\end{aligned}
$$

with

$$
\begin{aligned}
& \hat{\mathbf{Q}}_{\mathrm{LS}_{\mathrm{x} 0}}=\left[\begin{array}{cc}
2 \mathbf{Q}_{\mathrm{LS}_{\mathrm{x} 0}} & \mathbf{0} \\
\mathbf{0} & \mathbf{0}
\end{array}\right], \\
& \hat{\mathbf{Q}}_{\mathrm{LS} \mathrm{S}_{\mathrm{x}}}=\left[\begin{array}{cc}
\mathbf{0} & \mathbf{0} \\
\mathbf{0} & 2 \mathbf{Q}_{\mathrm{LS} \mathrm{x} 1}
\end{array}\right],
\end{aligned}
$$




$$
\begin{aligned}
& \hat{\mathbf{P}}_{\mathrm{LS}_{\mathrm{x}}}=\left[\begin{array}{cc}
\mathbf{P}_{\mathrm{LS} \mathrm{S}_{\mathrm{x}}} & \mathbf{0} \\
\mathbf{0} & \mathbf{P}_{\mathrm{LS} \mathrm{S}_{\mathrm{x}}}
\end{array}\right] \text {, } \\
& \hat{\mathbf{z}}_{\mathrm{LS} \mathrm{x}_{\mathrm{x}}}=\left[\begin{array}{c}
\mathbf{z}_{\mathrm{LS}} \mathbf{x}_{\mathrm{x} 0} \\
\mathbf{0}
\end{array}\right], \hat{\mathbf{z}}_{\mathrm{LS} \mathrm{S}_{\mathrm{x} 1}}=\left[\begin{array}{c}
\mathbf{0} \\
\mathbf{z}_{\mathrm{LS} \mathrm{S}_{\mathrm{x}}}
\end{array}\right],
\end{aligned}
$$

where 0 in (49) is an $N_{s} \times 1$ null matrix.

3) Given the obtained values of $a_{0,0}, a_{0,1}, a_{1,0}$ and $a_{1,1}$ in step 2), the new set of values of $\mathbf{w}_{\mathrm{A}}$ can be obtained by (44).

4) Repeat the steps of 2) and 3) until the cost function converges, i.e., the change of the cost function between the $\mathrm{k}$-th and $(\mathrm{k}+1)$-th iteration satisfies

$$
\left\|J_{\mathrm{LS}}(k+1)-J_{\mathrm{LS}}(k)\right\|_{2} \leq \delta\left\|J_{\mathrm{LS}}(k)\right\|_{2},
$$

where $\delta$ is a preset threshold value.

5) The final digital coefficients $\mathbf{w}_{\mathrm{D}, 0}, \mathbf{w}_{\mathrm{D}, 1}$ and the corresponding analogue coefficients $\mathbf{w}_{\mathrm{A}}$ are then obtained.

The convergence of the above iterative process is guaranteed. To see this, we define $\mathbf{w}_{\mathrm{D}}=\left[\mathbf{w}_{\mathrm{D}, 0} \mathbf{w}_{\mathrm{D}, 1}\right]$ and use $J_{\mathrm{LS}}\left(\mathbf{w}_{\mathrm{A}}, \mathbf{w}_{\mathrm{D}}\right)$ to represent the whole cost function. An important property of the cost function is that when $\mathbf{w}_{\mathrm{A}}$ is fixed, $J_{\mathrm{LS}}\left(\mathbf{w}_{\mathrm{A}}, \mathbf{w}_{\mathrm{D}}\right)$ is a convex function, while when $\mathbf{w}_{\mathrm{D}}$ is fixed, $J_{\mathrm{LS}}\left(\mathbf{w}_{\mathrm{A}}, \mathbf{w}_{\mathrm{D}}\right)$ is a convex function. As a result, at each iteration, given an optimized $\mathbf{w}_{D}$ in the last round, the newly optimized $\mathbf{w}_{\mathrm{A}}$ will at least not increase the value of the cost function, while given an optimized $\mathbf{w}_{\mathrm{A}}$ in the last round, the newly optimized $\mathbf{w}_{\mathrm{D}}$ will at least not increase the value of the cost function, i.e., the cost function will not increase during the alternate optimisation process.

ii) Three beams

In this section, consider the case of $M=X=3$. The steering vector of the $m$-th interleaved subarray is given by (21) with $m=\{0,1,2\}$. Then, similar to (24), the coefficients of the general digital coding scheme for the beam in direction $\varphi_{x}$ are

$$
\mathbf{w}_{\mathrm{D}, \mathrm{x}}=\left[w_{\mathrm{D}, \mathrm{x}, 0}, w_{\mathrm{D}, \mathrm{x}, 1}, w_{\mathrm{D}, \mathrm{x}, 2}\right]=\left[\begin{array}{lll}
a_{x, 0} & a_{x, 1} & a_{x, 2}
\end{array}\right],
$$

with $x=\{0,1,2\}$. Thus, the designed beam response for the beam pointing to direction $\varphi_{x}$ changes to

$$
\begin{aligned}
P_{\varphi_{\mathbf{x}}}(\theta) & =a_{x, 0} P_{0}(\theta)+a_{x, 1} P_{1}(\theta)+a_{x, 2} P_{2}(\theta) \\
& =a_{x, 0} \mathbf{w}_{0}^{\mathrm{H}} \mathbf{s}_{0}(\theta)+a_{x, 1} \mathbf{w}_{1}^{\mathrm{H}} \mathbf{s}_{1}(\theta)+a_{x, 2} \mathbf{w}_{2}^{\mathrm{H}} \mathbf{s}_{2}(\theta) .
\end{aligned}
$$

Similar to (29), the cost function $J_{\mathrm{LS}}$ combining the above three beams can be expanded as

$$
\begin{aligned}
& J_{\mathrm{LS}}= \\
& \sum_{x=0}^{X-1}\left(a_{x, 0}^{2} \mathbf{w}_{0}^{\mathrm{H}} \mathbf{Q}_{\mathrm{LS}} \mathbf{w}_{\mathbf{x} 0}+a_{x, 1}^{2} \mathbf{w}_{1}^{\mathrm{H}} \mathbf{Q}_{\mathrm{LS}} \mathbf{w}_{\mathbf{x}}+a_{x, 2}^{2} \mathbf{w}_{2}^{\mathrm{H}} \mathbf{Q}_{\mathrm{LS}} \mathbf{w}_{\mathbf{2}}\right. \\
& +a_{x, 0} a_{x, 1}\left(\mathbf{w}_{0}^{\mathrm{H}} \mathbf{P}_{\mathrm{LS}_{01 \mathbf{x}}} \mathbf{w}_{1}+\mathbf{w}_{1}^{\mathrm{H}} \mathbf{P}_{\mathrm{LS}_{10 \mathbf{x}}} \mathbf{w}_{0}\right) \\
& +a_{x, 0} a_{x, 2}\left(\mathbf{w}_{0}^{\mathrm{H}} \mathbf{P}_{\mathrm{LS}_{02 \mathbf{x}}} \mathbf{w}_{2}+\mathbf{w}_{2}^{\mathrm{H}} \mathbf{P}_{\mathrm{LS}_{20 \mathbf{x}}} \mathbf{w}_{0}\right) \\
& +a_{x, 1} a_{x, 2}\left(\mathbf{w}_{1}^{\mathrm{H}} \mathbf{P}_{\mathrm{LS}_{12 \mathrm{x}}} \mathbf{w}_{2}+\mathbf{w}_{2}^{\mathrm{H}} \mathbf{P}_{\mathrm{LS}_{21 \mathrm{x}}} \mathbf{w}_{1}\right) \\
& -a_{x, 0}\left(\mathbf{w}_{0}^{\mathrm{H}} \mathbf{z}_{\mathrm{LS}}{ }_{\mathrm{x} 0}+\mathbf{z}_{\mathrm{LS} \times 0}^{\mathrm{H}} \mathbf{w}_{0}\right)-a_{x, 1}\left(\mathbf{w}_{1}^{\mathrm{H}} \mathbf{z}_{\mathrm{LS} \mathrm{x}_{\mathbf{x}}}+\mathbf{z}_{\mathrm{LS} \mathrm{x}_{\mathrm{x}}}^{\mathrm{H}} \mathbf{w}_{1}\right) \\
& \left.-a_{x, 2}\left(\mathbf{w}_{2}^{\mathrm{H}} \mathbf{z}_{\mathrm{LS} \mathrm{x}_{2}}+\mathbf{z}_{\mathrm{LS} \mathbf{x} 2}^{\mathrm{H}} \mathbf{w}_{2}\right)+d_{\mathrm{LS}_{\mathrm{x}}}\right) \text {, }
\end{aligned}
$$

with

$$
\mathbf{P}_{\mathrm{LS} \mathrm{S}_{\mathbf{i k x}}}=(1-\beta) \sum_{\theta \in \Theta_{m_{\mathbf{x}}}} \mathbf{s}_{\mathbf{i}}(\theta) \mathbf{s}_{\mathbf{k}}(\theta)^{\mathrm{H}}+\frac{\beta}{N_{g_{\theta}}} \sum_{\theta \in \Theta_{s_{\mathbf{x}}}} \mathbf{s}_{\mathbf{i}}(\theta) \mathbf{s}_{\mathbf{k}}(\theta)^{\mathrm{H}},
$$

$\mathbf{Q}_{\mathrm{LS}_{\mathrm{xq}}}, \mathbf{z}_{\mathrm{LS}_{\mathrm{xq}}}, d_{\mathrm{LS}_{\mathrm{x}}}$ and $\mathbf{S}_{\mathrm{q}}(\theta)$ are the same as (30), (33), (34) and (35) with $\{q, i, k\}=\{0,1,2\}$ but $i \neq k$. Similar to (36), via combining the analogue coefficients $\mathbf{w}_{0}, \mathbf{w}_{1}$ and $\mathbf{w}_{2}$ into one vector, given by

$$
\mathbf{w}_{\mathrm{A}}=\left[\begin{array}{l}
\mathbf{w}_{0} \\
\mathbf{w}_{1} \\
\mathbf{w}_{2}
\end{array}\right],
$$

equation (53) can be rewritten as

$$
\begin{aligned}
J_{\mathrm{LS}} & =\mathbf{w}_{\mathrm{A}}^{\mathrm{H}}\left(\mathbf{Q}_{\mathrm{LS}}+\mathbf{P}_{\mathrm{LS}} \tilde{\mathbf{I}}_{\mathrm{LS}_{0}}+\mathbf{R}_{\mathrm{LS}} \tilde{\mathbf{I}}_{\mathrm{LS}}\right) \mathbf{w}_{\mathrm{A}}-\mathbf{w}_{\mathrm{A}}^{\mathrm{H}} \mathbf{z}_{\mathrm{LS}} \\
& -\mathbf{z}_{\mathrm{LS}}^{\mathrm{H}} \mathbf{w}_{\mathrm{A}}+d_{\mathrm{LS} 0}+d_{\mathrm{LS} 1}+d_{\mathrm{LS} 2}
\end{aligned}
$$

with

$$
\begin{aligned}
& \mathbf{Q}_{\mathrm{LS}}=\left[\begin{array}{ccc}
\mathrm{G}_{\mathrm{LS}_{0}} & \mathbf{0} & \mathbf{0} \\
\mathbf{0} & \mathbf{G}_{\mathrm{LS}_{1}} & \mathbf{0} \\
\mathbf{0} & \mathbf{0} & \mathbf{G}_{\mathrm{LS}_{2}}
\end{array}\right] \\
& \mathbf{P}_{\mathrm{LS}}=\left[\begin{array}{ccc}
\mathbf{H}_{\mathrm{LS}_{0}} & \mathbf{0} & \mathbf{0} \\
\mathbf{0} & \mathbf{H}_{\mathrm{LS}_{1}} & \mathbf{0} \\
\mathbf{0} & \mathbf{0} & \mathbf{H}_{\mathrm{LS}_{2}}
\end{array}\right] \text {, } \\
& \mathbf{R}_{\mathrm{LS}}=\left[\begin{array}{ccc}
\mathbf{Y}_{\mathrm{LS}_{0}} & \mathbf{0} & \mathbf{0} \\
\mathbf{0} & \mathbf{Y}_{\mathrm{LS}_{1}} & \mathbf{0} \\
\mathbf{0} & \mathbf{0} & \mathbf{Y}_{\mathrm{LS}_{2}}
\end{array}\right] \text {, } \\
& \tilde{\mathbf{I}}_{L S_{0}}=\left[\begin{array}{lll}
0 & 1 & 0 \\
0 & 0 & 1 \\
1 & 0 & 0
\end{array}\right] \\
& \tilde{\mathbf{I}}_{\mathrm{LS}_{1}}=\left[\begin{array}{lll}
\mathbf{0} & \mathbf{0} & \mathbf{1} \\
\mathbf{1} & \mathbf{0} & \mathbf{0} \\
\mathbf{0} & \mathbf{1} & \mathbf{0}
\end{array}\right] \text {, } \\
& \mathbf{z}_{\mathrm{LS}}=\left[\begin{array}{c}
a_{0,0} \mathbf{z}_{\mathrm{LS}}+a_{1,0} \mathbf{z}_{\mathrm{LS}}+a_{2,0} \mathbf{z}_{\mathrm{LS}} \\
a_{0,1} \mathbf{z}_{\mathrm{LS}}+a_{1,1} \mathbf{z}_{\mathrm{LS}}+a_{2,1} \mathbf{z}_{\mathrm{LS}} \\
a_{0,2} \mathbf{z}_{\mathrm{LS}}+a_{1,2} \mathbf{z}_{\mathrm{LS}}+a_{2,2} \mathbf{z}_{\mathrm{LS}_{22}}
\end{array}\right], \\
& \mathbf{G}_{\mathrm{LS}_{\mathrm{q}}}=a_{0, \mathrm{q}}^{2} \mathbf{Q}_{\mathrm{LS}}+a_{1, \mathrm{q}}^{2} \mathbf{Q}_{\mathrm{LS}_{1 \mathrm{q}}}+a_{2, \mathrm{q}}^{2} \mathbf{Q}_{\mathrm{LS}}, \\
& \mathbf{H}_{\mathrm{LS}_{0}}=a_{0,0} a_{0,1} \mathbf{P}_{\mathrm{LS}_{010}}+a_{1,0} a_{1,1} \mathbf{P}_{\mathrm{LS}_{011}}+a_{2,0} a_{2,1} \mathbf{P}_{\mathrm{LS}_{012}}, \\
& \mathbf{H}_{\mathrm{LS}}=a_{0,1} a_{0,2} \mathbf{P}_{\mathrm{LS}_{120}}+a_{1,1} a_{1,2} \mathbf{P}_{\mathrm{LS}_{121}}+a_{2,1} a_{2,2} \mathbf{P}_{\mathrm{LS}_{122}} \text {, } \\
& \mathbf{H}_{\mathrm{LS}_{2}}=a_{0,0} a_{0,2} \mathbf{P}_{\mathrm{LS}_{200}}+a_{1,0} a_{1,2} \mathbf{P}_{\mathrm{LS}_{201}}+a_{2,0} a_{2,2} \mathbf{P}_{\mathrm{LS}_{202}} \text {, } \\
& \mathbf{Y}_{\mathrm{LS}_{0}}=a_{0,0} a_{0,2} \mathbf{P}_{\mathrm{LS}_{020}}+a_{1,0} a_{1,2} \mathbf{P}_{\mathrm{LS}_{021}}+a_{2,0} a_{2,2} \mathbf{P}_{\mathrm{LS}_{022}}, \\
& \mathbf{Y}_{\mathrm{LS}_{1}}=a_{0,0} a_{0,1} \mathbf{P}_{\mathrm{LS}_{100}}+a_{1,0} a_{1,1} \mathbf{P}_{\mathrm{LS}_{101}}+a_{2,0} a_{2,1} \mathbf{P}_{\mathrm{LS}_{102}}, \\
& \mathbf{Y}_{\mathrm{LS}_{2}}=a_{0,1} a_{0,2} \mathbf{P}_{\mathrm{LS}_{210}}+a_{1,1} a_{1,2} \mathbf{P}_{\mathrm{LS}_{211}}+a_{2,1} a_{2,2} \mathbf{P}_{\mathrm{LS}_{212}} \text {. }
\end{aligned}
$$

Similar to (44), for a given set of $a_{\mathrm{x}, \mathrm{q}}(\{x, q\} \in\{0,1,2\}), \mathbf{w}_{\mathrm{A}}$ can be obtained by

$$
\mathbf{w}_{\mathrm{A}}=\left(\mathbf{Q}_{\mathrm{LS}}+\mathbf{P}_{\mathrm{LS}} \tilde{\mathbf{I}}_{\mathrm{LS}}+\mathbf{R}_{\mathrm{LS}} \tilde{\mathbf{I}}_{\mathrm{LS}}\right)^{-1} \mathbf{z}_{\mathrm{LS}}
$$

Joint optimisation of the digital coefficients $a_{\mathrm{x}, \mathrm{q}}(\{x, q\} \in$ $\{0,1,2\})$ and the corresponding analogue coefficients $\mathbf{w}_{\mathrm{A}}$ can be achieved by the following iterative process: 
1) First, via initialising the digital coefficients $a_{0,0}=$ $a_{0,1}=a_{1,0}=a_{1,2}=a_{2,0}=a_{2,1}=a_{2,2}=1$ and $a_{0,2}=a_{1,1}=-1$, the values of $\mathbf{w}_{\mathrm{A}}$ are obtained by substituting $a_{\mathrm{x}, \mathrm{q}}(\{x, q\} \in\{0,1,2\})$ into (66).

2) Given the obtained values of $\mathbf{w}_{\mathrm{A}}$ in step 1), we find the closed-form solution of digital coefficients $a_{\mathrm{x}, \mathrm{q}}(\{x, q\} \in$ $\{0,1,2\})$ by minimising the cost function (53), given by

$$
\begin{aligned}
& {\left[\begin{array}{l}
a_{\mathrm{x}, 0} \\
a_{\mathrm{x}, 1} \\
a_{\mathrm{x}, 2}
\end{array}\right]=}
\end{aligned}
$$

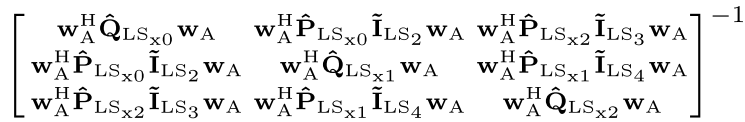

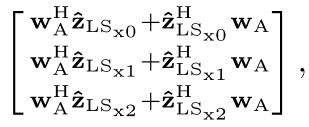

with

$$
\begin{aligned}
& \hat{\mathbf{Q}}_{\mathrm{LS}_{\mathrm{x} 0}}=\left[\begin{array}{ccc}
2 \mathbf{Q}_{\mathrm{LS}_{\mathrm{x} 0}} & \mathbf{0} & \mathbf{0} \\
\mathbf{0} & \mathbf{0} & \mathbf{0} \\
\mathbf{0} & \mathbf{0} & \mathbf{0}
\end{array}\right] \\
& \hat{\mathbf{Q}}_{\mathrm{LS} \mathrm{x}_{\mathbf{1}}}=\left[\begin{array}{ccc}
\mathbf{0} & \mathbf{0} & \mathbf{0} \\
\mathbf{0} & 2 \mathbf{Q}_{\mathrm{LS} \times 1} & \mathbf{0} \\
\mathbf{0} & \mathbf{0} & \mathbf{0}
\end{array}\right] \text {, } \\
& \hat{\mathbf{Q}}_{\mathrm{LS}_{\mathrm{x} 2}}=\left[\begin{array}{ccc}
\mathbf{0} & \mathbf{0} & \mathbf{0} \\
\mathbf{0} & \mathbf{0} & \mathbf{0} \\
\mathbf{0} & \mathbf{0} & 2 \mathbf{Q}_{\mathrm{LS}_{\mathrm{x} 2}}
\end{array}\right] \text {, } \\
& \hat{\mathbf{P}}_{\mathrm{LS}_{\mathrm{x} 0}}=\left[\begin{array}{ccc}
\mathbf{P}_{\mathrm{LS}_{01 \mathrm{x}}} & \mathbf{0} & \mathbf{0} \\
\mathbf{0} & \mathbf{P}_{\mathrm{LS}_{10 \mathrm{x}}} & \mathbf{0} \\
\mathbf{0} & \mathbf{0} & \mathbf{0}
\end{array}\right] \text {, } \\
& \hat{\mathbf{P}}_{\mathrm{LS}_{\mathrm{x} 1}}=\left[\begin{array}{ccc}
\mathbf{0} & \mathbf{0} & \mathbf{0} \\
\mathbf{0} & \mathbf{P}_{\mathrm{LS}_{12 \mathrm{x}}} & \mathbf{0} \\
\mathbf{0} & \mathbf{0} & \mathbf{P}_{\mathrm{LS}_{21 \mathrm{x}}}
\end{array}\right] \text {, } \\
& \hat{\mathbf{P}}_{\mathrm{LS}_{\mathrm{x} 2}}=\left[\begin{array}{ccc}
\mathbf{P}_{\mathrm{LS}_{02 \mathrm{x}}} & \mathbf{0} & \mathbf{0} \\
\mathbf{0} & \mathbf{0} & \mathbf{0} \\
\mathbf{0} & \mathbf{0} & \mathbf{P}_{\mathrm{LS}_{20 \mathrm{x}}}
\end{array}\right] \text {, } \\
& \tilde{\mathbf{I}}_{\mathrm{LS}_{2}}=\left[\begin{array}{lll}
0 & 1 & 0 \\
1 & 0 & 0 \\
0 & 0 & 1
\end{array}\right] \text {, } \\
& \tilde{\mathbf{I}}_{\mathrm{LS}_{3}}=\left[\begin{array}{lll}
0 & 0 & 1 \\
0 & 1 & 0 \\
1 & 0 & 0
\end{array}\right] \text {, } \\
& \tilde{\mathbf{I}}_{\mathrm{LS}_{4}}=\left[\begin{array}{lll}
1 & 0 & 0 \\
\mathbf{0} & \mathbf{0} & \mathbf{1} \\
\mathbf{0} & \mathbf{1} & \mathbf{0}
\end{array}\right] \text {, } \\
& \hat{\mathbf{z}}_{\mathrm{LS}_{\mathrm{x} 0}}=\left[\begin{array}{c}
\mathbf{z}_{\mathrm{LS}} \\
\mathbf{0} \\
\mathbf{0}
\end{array}\right], \hat{\mathbf{z}}_{\mathrm{LS} \mathrm{x}_{1}}=\left[\begin{array}{c}
\mathbf{0} \\
\mathbf{z}_{\mathrm{LS}} \\
\mathbf{0}
\end{array}\right], \hat{\mathbf{z}}_{\mathrm{LS} \mathrm{x}_{\mathrm{x}}}=\left[\begin{array}{c}
\mathbf{0} \\
\mathbf{0} \\
\mathbf{z}_{\mathrm{LS} \mathrm{S}_{\mathrm{x}}} \\
(77)
\end{array}\right.
\end{aligned}
$$

Note that 0 in (77) is an $N_{s} \times 1$ null matrix.

3) Given the obtained values of $a_{\mathrm{x}, \mathrm{q}}(\{x, q\} \in\{0,1,2\})$ in step 2), the new set of values of $\mathbf{w}_{\mathrm{A}}$ can be obtained by (66).
4) Repeat the steps of 2) and 3) until the cost function converges and the final digital coefficients $\mathbf{w}_{\mathrm{D}, 0}, \mathbf{w}_{\mathrm{D}, 1}$, $\mathbf{w}_{\mathrm{D}, 2}$ and the corresponding analogue coefficients $\mathbf{w}_{\mathrm{A}}$ are then obtained.

\section{iii) More than three beams}

Following the approach introduced for two beams and three beams, we can extend it to more than three beams without difficulty. To save space, it is omitted here.

\section{B. Uniform Planar Array}

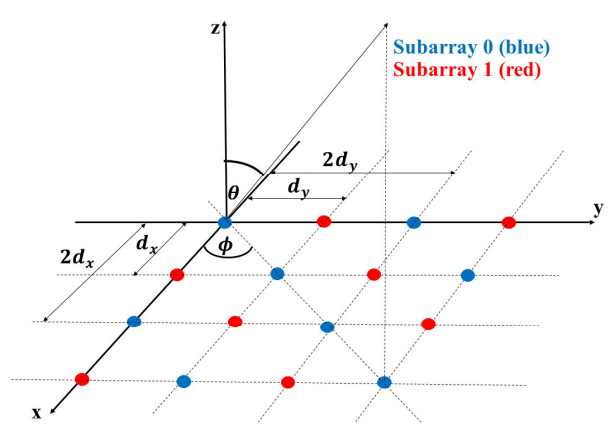

Fig. 2. A UPA with interleaved subarray architecture.

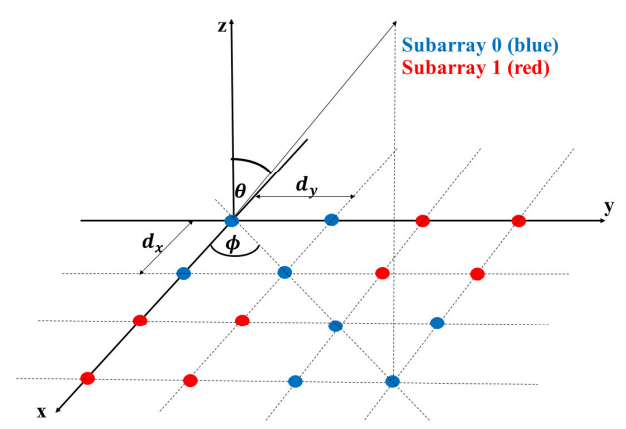

Fig. 3. A UPA with localised subarray architecture.

The approach introduced for designing uniform linear arrays can be extended to uniform planar arrays too and the only thing to change is the steering vector and the desired beam response, as for planar arrays, both elevation angle and azimuth angle are needed to specify a beam response in the three dimensional space [16], [31], [32].

Similar to the linear array case, for hybrid beamforming based on a UPA, there are also two different structures, i.e., interleaved and localised, and an example for each case with $M=2$ are displayed in Figs. 2 and 3, which contain $2 N_{x}$ and $2 N_{y}$ antennas along the $\mathrm{x}$-axis and $\mathrm{y}$-axis, respectively. The adjacent antenna spacings along the $\mathrm{x}$-axis and $\mathrm{y}$-axis are $d_{x}$ and $d_{y}$. Moreover, the elevation angle is $\theta \in\left[-90^{\circ}, 90^{\circ}\right]$ and azimuth angle is $\phi \in\left[-90^{\circ}, 90^{\circ}\right]$. Thus, the steering vectors of the two interleaved subarrays as a function of $\theta$ and $\phi$ are 
given by

$$
\begin{aligned}
& \mathbf{s}_{0}(\theta, \phi)= \\
& {\left[1, e^{j 2 \pi \frac{2 d_{y}}{\lambda} \sin \theta \sin \phi}, \ldots, e^{j 2 \pi \frac{2\left(N_{y}-1\right) d_{y}}{\lambda} \sin \theta \sin \phi},\right.} \\
& e^{j 2 \pi\left(\frac{d_{x}}{\lambda} \sin \theta \cos \phi+\frac{d_{y}}{\lambda} \sin \theta \sin \phi\right)}, \ldots, \\
& e^{j 2 \pi\left(\frac{d_{x}}{\lambda} \sin \theta \cos \phi+\frac{\left(2 N_{y}-1\right) d_{y}}{\lambda} \sin \theta \sin \phi\right)}, \ldots, \\
& \left.e^{j 2 \pi\left(\frac{\left(2 N_{x}-1\right) d_{x}}{\lambda} \sin \theta \cos \phi+\frac{\left(2 N_{y}-1\right) d_{y}}{\lambda} \sin \theta \sin \phi\right)}\right]^{\mathrm{T}}, \\
& \mathbf{s}_{1}(\theta, \phi)= \\
& {\left[e^{j 2 \pi \frac{d_{y}}{\lambda} \sin \theta \sin \phi}, \ldots, e^{j 2 \pi \frac{\left(2 N_{y}-1\right) d_{y}}{\lambda} \sin \theta \sin \phi},\right.} \\
& e^{j 2 \pi \frac{d_{x}}{\lambda} \sin \theta \cos \phi}, \ldots, e^{j 2 \pi\left(\frac{d_{x}}{\lambda} \sin \theta \cos \phi+\frac{2\left(N_{y}-1\right) d_{y}}{\lambda} \sin \theta \sin \phi\right)}, \\
& \left.\ldots, e^{j 2 \pi\left(\frac{\left(2 N_{x}-1\right) d_{x}}{\lambda} \sin \theta \cos \phi+\frac{2\left(N_{y}-1\right) d_{y}}{\lambda} \sin \theta \sin \phi\right)}\right]^{\mathrm{T}} .
\end{aligned}
$$

If all antennas are equally spaced in $\mathrm{x}$ and $\mathrm{y}$ axes, i.e., $d_{x}=d_{y}=d$, the steering vectors of two subarrays in (78) change to

$$
\begin{aligned}
& \mathbf{s}_{0}(\theta, \phi)= \\
& {\left[1, e^{j 2 \pi \frac{2 d}{\lambda} \sin \theta \sin \phi}, \ldots, e^{j 2 \pi \frac{2\left(N_{y}-1\right) d}{\lambda} \sin \theta \sin \phi},\right.} \\
& e^{j 2 \pi \frac{d}{\lambda}(\sin \theta \cos \phi+\sin \theta \sin \phi)}, \ldots, \\
& e^{j 2 \pi \frac{d}{\lambda}\left(\sin \theta \cos \phi+\left(2 N_{y}-1\right) \sin \theta \sin \phi\right)}, \ldots, \\
& \left.e^{j 2 \pi \frac{d}{\lambda}\left(\left(2 N_{x}-1\right) \sin \theta \cos \phi+\left(2 N_{y}-1\right) \sin \theta \sin \phi\right)}\right]^{\mathrm{T}}, \\
& \mathbf{s}_{1}(\theta, \phi)= \\
& {\left[e^{j 2 \pi \frac{d}{\lambda} \sin \theta \sin \phi}, \ldots, e^{j 2 \pi \frac{\left(2 N_{y}-1\right) d}{\lambda} \sin \theta \sin \phi},\right.} \\
& e^{j 2 \pi \frac{d}{\lambda} \sin \theta \cos \phi}, \ldots, e^{j 2 \pi \frac{d}{\lambda}\left(\sin \theta \cos \phi+2\left(N_{y}-1\right) \sin \theta \sin \phi\right)}, \\
& \left.\ldots, e^{j 2 \pi \frac{d}{\lambda}\left(\left(2 N_{x}-1\right) \sin \theta \cos \phi+2\left(N_{y}-1\right) \sin \theta \sin \phi\right)}\right]^{\mathrm{T}} .
\end{aligned}
$$

Again, suppose the coefficients vector for the two subarrays are represented by $\mathbf{w}_{0}$ and $\mathbf{w}_{1}$, respectively. Then, similar to (36), by combining $\mathbf{w}_{0}$ and $\mathbf{w}_{1}$ into one vector $\mathbf{w}_{\mathrm{A}}$ and using the same approach as in Section IV-Ai), the final digital and analogue coefficients can be obtained for the UPA based hybrid beamforming structure.

\section{DESIGNED EXAMPLES}

In this section, some design examples are provided for the two proposed methods. Assume that each subarray consists of ten and fifteen antennas with ULA, i.e., $N_{s}=10$ and $N_{s}=15$ for the two-user and three-user cases, respectively. Moreover, for UPA, each subarray contains $N_{x}=N_{y}=6$ antennas along the $\mathrm{x}$ and $\mathrm{y}$ axes, respectively. The performance of the scheme in [26] and the two proposed designs in Sections III and IV are compared for multiple arbitrary directions.

\section{A. Design example with the scheme in [26]}

Suppose that the two desired beam directions are $-48^{\circ}$ and $20^{\circ}$. For the scheme in [26], the two-beam multiplexing performance of one desired beam pointing to $-48^{\circ}$ and one pointing to $20^{\circ}$ are shown in Fig. 4 and Fig. 5. The corresponding analogue coefficients are displayed in Table I and Table II, respectively.

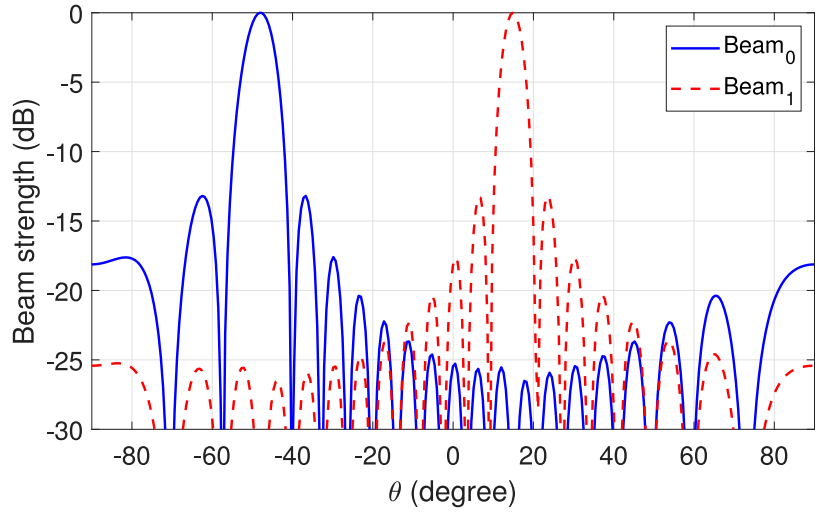

Fig. 4. Beam pattern of two interleaved subarrays when $\varphi_{0}=-48^{\circ}$ with the scheme in [26].

TABLE I

Analogue coefficients $w_{0}$ and $w_{1}$ with its zeroth beam pointing to $-48^{\circ}$ using the scheme in [26].

\begin{tabular}{|c|c|c|}
\hline $\mathrm{n}$ & $\mathbf{w}_{0}$ & $\mathbf{w}_{1}$ \\
\hline 0 & $1.0000+0.0000 \mathrm{i}$ & $0.6911-0.7228 \mathrm{i}$ \\
\hline 1 & $-0.0431-0.9991 \mathrm{i}$ & $-0.7530-0.6580 \mathrm{i}$ \\
\hline 2 & $-0.9963+0.0860 \mathrm{i}$ & $-0.6236+0.7817 \mathrm{i}$ \\
\hline 3 & $0.1289+0.9917 \mathrm{i}$ & $0.8089+0.5880 \mathrm{i}$ \\
\hline 4 & $0.9852-0.1714 \mathrm{i}$ & $0.5511-0.8344 \mathrm{i}$ \\
\hline 5 & $-0.2137-0.9769 \mathrm{i}$ & $-0.8583-0.5132 \mathrm{i}$ \\
\hline 6 & $-0.9668+0.2556 \mathrm{i}$ & $-0.4742+0.8804 \mathrm{i}$ \\
\hline 7 & $0.2970+0.9549 \mathrm{i}$ & $0.9008+0.4343 \mathrm{i}$ \\
\hline 8 & $0.9412-0.3378 \mathrm{i}$ & $0.3935-0.9193 \mathrm{i}$ \\
\hline 9 & $-0.3780-0.9258 \mathrm{i}$ & $-0.9360-0.3519 \mathrm{i}$ \\
\hline
\end{tabular}

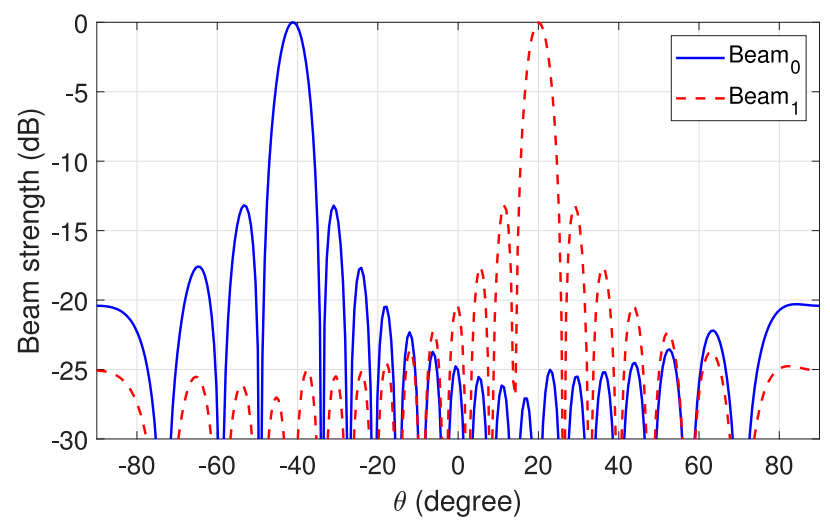

Fig. 5. Beam pattern of two interleaved subarrays when $\varphi_{1}=20^{\circ}$ with the scheme in [26].

We can clearly observe that in Fig. 4, the first beam has pointed to the direction $14.9^{\circ}$ instead of the required $20^{\circ}$ by the design, while in Fig. 5, the zeroth beam has pointed $-41.1^{\circ}$ instead of the required $-48^{\circ}$, highlighting the issue of the design in [26].

\section{B. Design example for the first proposed scheme}

For the first proposed design with varying antenna spacing, according to (20), $\alpha$ can be calculated as 0.92 for $\varphi_{0}=-48^{\circ}$ and $\varphi_{1}=20^{\circ}$. Thus, the adjacent antenna spacing for the two subarrays is $d_{0}=d_{1}=\alpha \lambda=0.92 \lambda$. The two resultant beams 
TABLE II

Analogue coefficients $w_{0}$ and $w_{1}$ with its first beam pointing to $20^{\circ}$ using the scheme in [26].

\begin{tabular}{|c|c|c|}
\hline $\mathrm{n}$ & $\mathbf{w}_{0}$ & $\mathbf{w}_{1}$ \\
\hline 0 & $1.0000+0.0000 \mathrm{i}$ & $0.4762-0.8793 \mathrm{i}$ \\
\hline 1 & $-0.5497-0.8354 \mathrm{i}$ & $-0.9967+0.0818 \mathrm{i}$ \\
\hline 2 & $-0.3957+0.9184 \mathrm{i}$ & $0.6132+0.7900 \mathrm{i}$ \\
\hline 3 & $0.9847-0.1742 \mathrm{i}$ & $0.3265-0.9452 \mathrm{i}$ \\
\hline 4 & $-0.6868-0.7268 \mathrm{i}$ & $-0.9700+0.2432 \mathrm{i}$ \\
\hline 5 & $-0.2296+0.9733 \mathrm{i}$ & $0.7337+0.6794 \mathrm{i}$ \\
\hline 6 & $0.9393-0.3432 \mathrm{i}$ & $0.1680-0.9858 \mathrm{i}$ \\
\hline 7 & $-0.8030-0.5960 \mathrm{i}$ & $-0.9174+0.3980 \mathrm{i}$ \\
\hline 8 & $-0.0565+0.9984 \mathrm{i}$ & $0.8347+0.5507 \mathrm{i}$ \\
\hline 9 & $0.8651-0.5016 \mathrm{i}$ & $0.0051-1.0000 \mathrm{i}$ \\
\hline
\end{tabular}

by the first proposed design with varying antenna spacing are displayed in Fig. 6. The corresponding analogue coefficients are listed in Table III. It is clear that the two beams are in the desired directions and the first proposed design is working effectively.

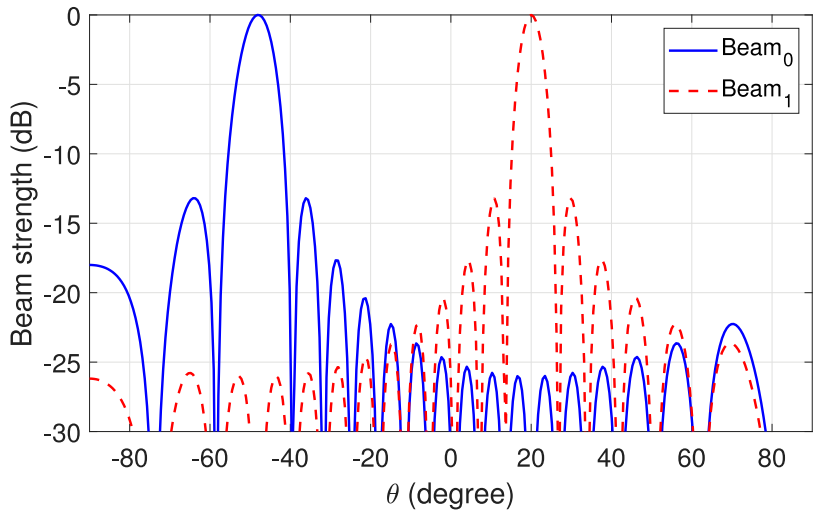

Fig. 6. Beam pattern of two interleaved subarrays when $\varphi_{0}=-48^{\circ}$ and $\varphi_{1}=20^{\circ}$ for the first proposed scheme in Section III.

TABLE III

Analogue coefficients $w_{0}$ and $w_{1}$ when $\varphi_{0}=-48^{\circ}$ and $\varphi_{1}=20^{\circ}$ for the first proposed scheme in Section III

\begin{tabular}{|c|c|c|}
\hline $\mathrm{n}$ & $\mathbf{w}_{0}$ & $\mathbf{w}_{1}$ \\
\hline 0 & $1.0000+0.0000 \mathrm{i}$ & $0.5486-0.8361 \mathrm{i}$ \\
\hline 1 & $-0.3982-0.9173 \mathrm{i}$ & $-0.9854-0.1703 \mathrm{i}$ \\
\hline 2 & $-0.6829+0.7305 \mathrm{i}$ & $0.2362+0.9717 \mathrm{i}$ \\
\hline 3 & $0.9420+0.3356 \mathrm{i}$ & $0.7973-0.6035 \mathrm{i}$ \\
\hline 4 & $-0.0673-0.9977 \mathrm{i}$ & $-0.8711-0.4911 \mathrm{i}$ \\
\hline 5 & $-0.8885+0.4590 \mathrm{i}$ & $-0.1036+0.9946 \mathrm{i}$ \\
\hline 6 & $0.7748+0.6322 \mathrm{i}$ & $0.9536-0.3010 \mathrm{i}$ \\
\hline 7 & $0.2715-0.9624 \mathrm{i}$ & $-0.6558-0.7549 \mathrm{i}$ \\
\hline 8 & $-0.9910+0.1342 \mathrm{i}$ & $-0.4314+0.9022 \mathrm{i}$ \\
\hline 9 & $0.5177+0.8556 \mathrm{i}$ & $0.9993+0.0365 \mathrm{i}$ \\
\hline
\end{tabular}

C. Design examples for the second proposed scheme based on ULA

As to the second proposed design using ULA, we consider two fixed antenna spacings $d=\frac{\lambda}{3}$ and $d=\frac{2 \lambda}{9}$ for the two-user and three-user cases, respectively.

\section{i) Two-user case}

For the two-user case, the trade-off factor in the weighting function is chosen as $\beta=0.65$ and the convergence factor is $\delta=1 \times 10^{-5}$. Because the sidelobe regions are all sampled at $1^{\circ}$ and the same beam directions as in the first proposed design are adopted, the mainlobe direction of the zeroth beam is $\Theta_{m_{0}}=-48^{\circ}$ with the sidelobe region $\Theta_{s_{0}} \in\left[-90^{\circ},-53^{\circ}\right] \cup\left[-43^{\circ}, 90^{\circ}\right]$, and the mainlobe direction of the first beam is $\Theta_{m_{1}}=20^{\circ}$ with the sidelobe region $\Theta_{s_{1}} \in\left[-90^{\circ}, 15^{\circ}\right] \cup\left[25^{\circ}, 90^{\circ}\right]$ and $N_{g}=172$.

With $d=\frac{\lambda}{3}$, the zeroth and first beams obtained using the scheme in Section IV ('2nd proposed method') are shown in Figs. 7 and 8, respectively. The corresponding digital and analogue coefficients are displayed in Tables IV and V and the change of the cost function $J_{\mathrm{LS}}$ with respect to the iteration number is shown in Fig. 9. Furthermore, we also showed the design results obtained using the method in Appendix, where the zeroth subarray in the interleaved structure is directly used to design a beam pointing to $-48^{\circ}$ and the first subarray for the beam pointing to $20^{\circ}$; there are no digital schemes combining these two subarrays and each subarray operates independent of the other. They are indicated in Figs. 7 and 8 as 'Separate direct design 0 '. Moreover, we have also showed the separate design results using the method in Appendix based on the localised subarray structure, i.e., for the whole ULA with $2 N_{s}$ sensors, the first $N_{s}$ of them are used to design the bream pointing to $-48^{\circ}$ and the last $N_{s}$ of them are used to design the beam pointing to $20^{\circ}$; there is no digital inter-subarray coding schemes to combine these two together. This result is represented by 'Separate direct design 1 ' in Figs. 7 and 8.

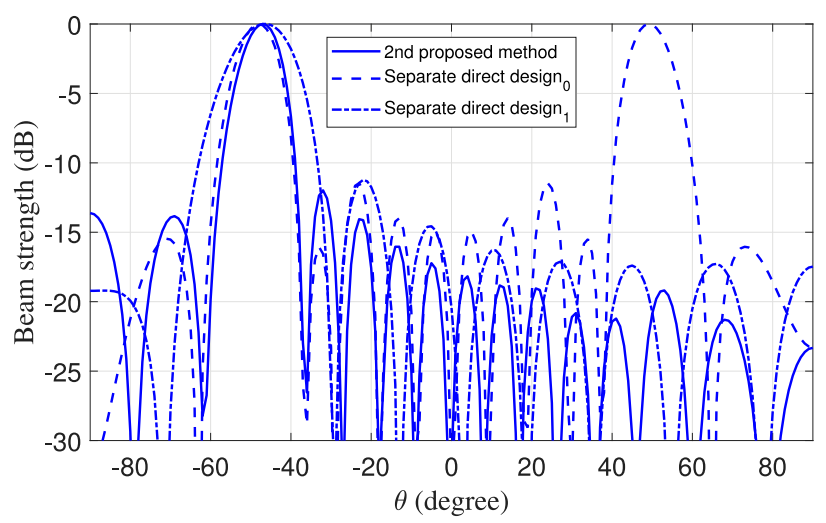

Fig. 7. Beam pattern of the zeroth beam when $\varphi_{0}=-48^{\circ}$ for the second proposed scheme in Section IV-Ai) and the separate direct designs with the interleaved and localised architectures using the method in Appendix $(d=$ $\left.\frac{1}{3} \lambda\right)$ 


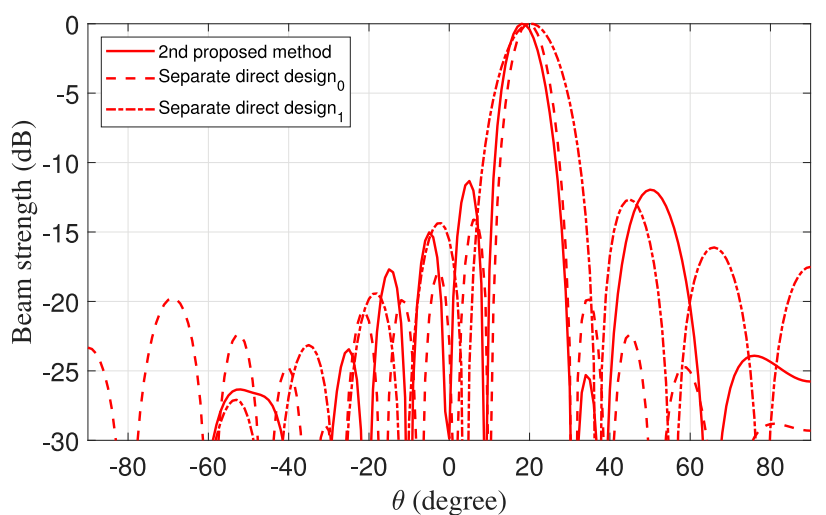

Fig. 8. Beam pattern of the first beam when $\varphi_{1}=20^{\circ}$ for the second proposed scheme in Section IV-Ai) and the separate direct designs with the interleaved and localised architectures using the method in Appendix $(d=$ $\left.\frac{1}{3} \lambda\right)$.

\section{TABLE IV}

Digital coefficients $\mathbf{w}_{\mathrm{D}, 0}$ and $\mathbf{w}_{\mathrm{D}, 1}$ when $\varphi_{0}=-48^{\circ}$ and $\varphi_{1}=20^{\circ}$ for the second proposed scheme in Section IV-Ai) $\left(d=\frac{1}{3} \lambda\right)$.

\begin{tabular}{|c|c|c|}
\hline $\mathrm{m}$ & $\mathbf{w}_{\mathrm{D}, 0}$ & $\mathbf{w}_{\mathrm{D}, 1}$ \\
\hline 0 & 2.3775 & 0.7983 \\
\hline 1 & -1.5846 & 0.3237 \\
\hline
\end{tabular}

TABLE V

Analogue coefficients $\mathbf{w}_{0}$ and $\mathbf{w}_{1}$ when $\varphi_{0}=-48^{\circ}$ and $\varphi_{1}=20^{\circ}$ for the second proposed scheme in Section IV-Ai) $\left(d=\frac{1}{3} \lambda\right)$.

\begin{tabular}{|c|c|c|}
\hline $\mathrm{n}$ & $\mathrm{w}$ & $\mathbf{w}_{0}$ \\
\hline 0 & $0.0515+0.0411 \mathrm{i}$ & $0.0263+0.1422 \mathrm{i}$ \\
\hline 1 & $-0.0297+0.0886 \mathrm{i}$ & $-0.0793+0.0753 \mathrm{i}$ \\
\hline 2 & $-0.0638+0.0192 \mathrm{i}$ & $-0.1161-0.0371 \mathrm{i}$ \\
\hline 3 & $-0.0533-0.0901 \mathrm{i}$ & $0.0458-0.1666 \mathrm{i}$ \\
\hline 4 & $0.1000-0.0467 \mathrm{i}$ & $0.1349+0.0547 \mathrm{i}$ \\
\hline 5 & $0.0412+0.0683 \mathrm{i}$ & $0.0051+0.1066 \mathrm{i}$ \\
\hline 6 & $-0.0399+0.0723 \mathrm{i}$ & $-0.1311+0.0543 \mathrm{i}$ \\
\hline 7 & $-0.0853-0.0404 \mathrm{i}$ & $-0.0340-0.1297 \mathrm{i}$ \\
\hline 8 & $0.0320-0.0692 \mathrm{i}$ & $0.0515-0.0600 \mathrm{i}$ \\
\hline 9 & $0.0249-0.0367 \mathrm{i}$ & $0.0531-0.0413 \mathrm{i}$ \\
\hline
\end{tabular}

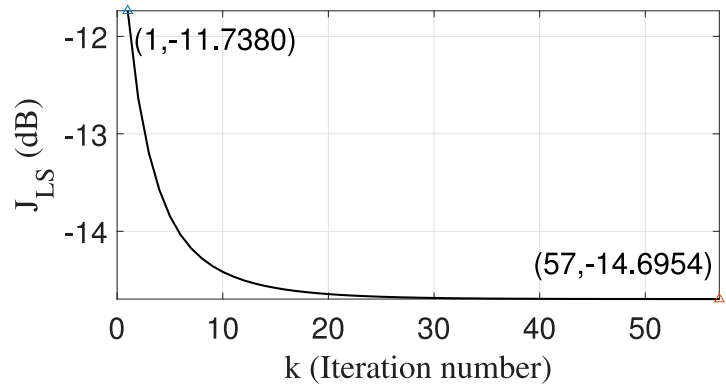

Fig. 9. Cost function $J_{\mathrm{LS}}$ with respect to the iteration number $k$ for the two-user case when $\varphi_{0}=-48^{\circ}$ and $\varphi_{1}=20^{\circ}$ with two ULAs $\left(d=\frac{1}{3} \lambda\right)$.

For the deign example in Fig. 8, although the improvement for the first beam is not prominent enough compared to the design by 'Separate direct design 0 ', the quality of the zeroth beam in Fig. 7 by the second proposed scheme is much better than the two separate direct designs, as the sidelobes have been suppressed to a much lower level compared to the 'Separate direct design,', while its mainlobe beamwidth is much narrower than that of the 'Separate direct design ${ }_{1}$ '.

In addition, to show the effect of the constraint imposed by the hybrid beamforming structure, we design the zeroth beam using the whole array directly using the method in Appendix (without inter-subarray coding and without the hybrid structure, just a classic beamformer with the same number of coefficients as the number of antennas) and then design the first beam using the whole array in the same way. With the same other parameters, the two resultant beams designed by the second proposed method and the design based on the whole array ('Total design') are compared in Fig. 10.

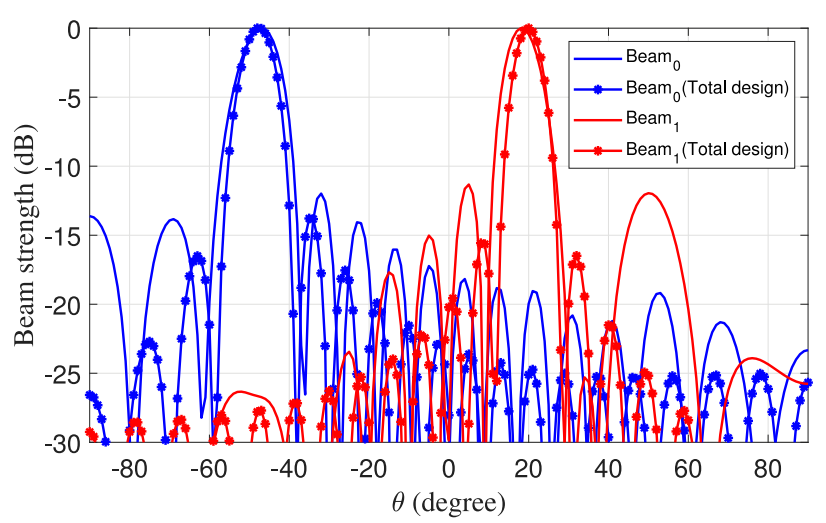

Fig. 10. Beam pattern of the two designed beams when $\varphi_{0}=-48^{\circ}$ and $\varphi_{1}=20^{\circ}$ for the second proposed scheme in Section IV-Ai) and the total separate direct design in Appendix $\left(d=\frac{1}{3} \lambda\right)$.

As clearly shown in Fig. 10, the mainlobe beamwidths of the two designed beams in 'Total design' are narrower than those of the second proposed scheme, in addition to a much lower sidelobe level, as more degrees of freedom are available by removing the constraint of the hybrid beamforming structure.

\section{ii) Three-user case}

Now, we consider examples for the three-user case. Suppose that the three desired beam directions are $-55^{\circ},-5^{\circ}$ and $40^{\circ}$, the corresponding sidelobe regions are $\Theta_{s_{0}} \in\left[-90^{\circ},-60^{\circ}\right] \cup$ $\left[-50^{\circ}, 90^{\circ}\right], \Theta_{s_{1}} \in\left[-90^{\circ},-10^{\circ}\right] \cup\left[0^{\circ}, 90^{\circ}\right]$ and $\Theta_{s_{2}} \in$ $\left[-90^{\circ}, 35^{\circ}\right] \cup\left[45^{\circ}, 90^{\circ}\right]$, and $N_{g}=172$. The trade-off factor is $\beta=0.7$ and the convergence factor changes to $\delta=1 \times 10^{-4}$.

With $d=\frac{2}{9} \lambda$, the three resultant beams are shown in Figs. 11, 12 and 13 and the corresponding digital and analogue coefficients are listed in Tables VI and VII, respectively. A similar observation can be made as in the two-beam case. 


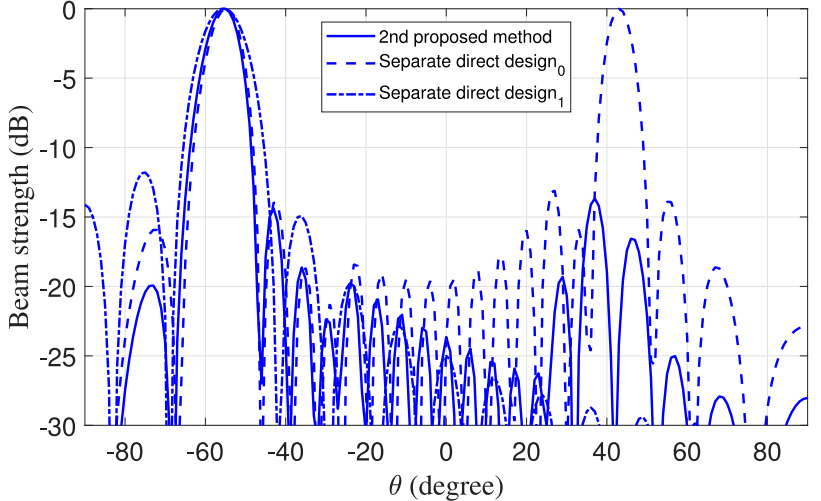

Fig. 11. Beam pattern of the zeroth beam with $\varphi_{0}=-55^{\circ}$ for the second proposed scheme in Section IV-Aii) and the separate direct designs with the interleaved and localised architectures using the method in Appendix $(d=$ $\left.\frac{2}{9} \lambda\right)$.

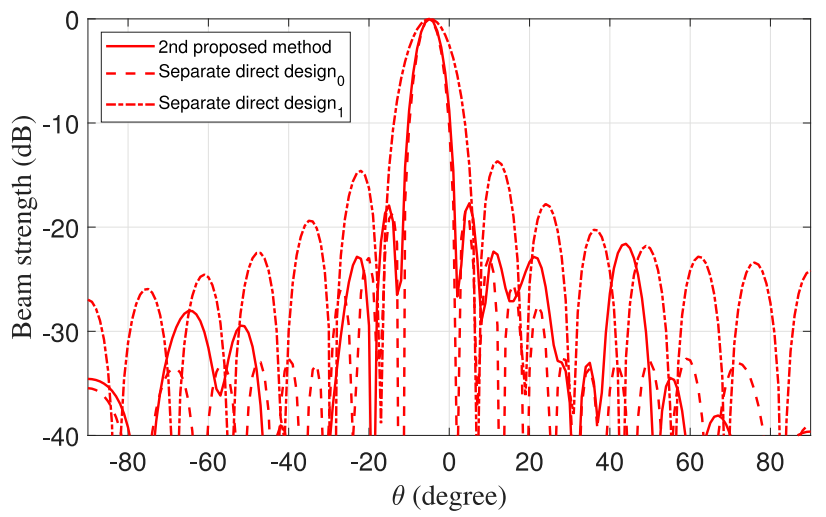

Fig. 12. Beam pattern of the first beam with $\varphi_{1}=-5^{\circ}$ for the second proposed scheme in Section IV-Aii) and the separate direct designs with the interleaved and localised architectures using the method in Appendix $(d=$ $\left.\frac{2}{9} \lambda\right)$.

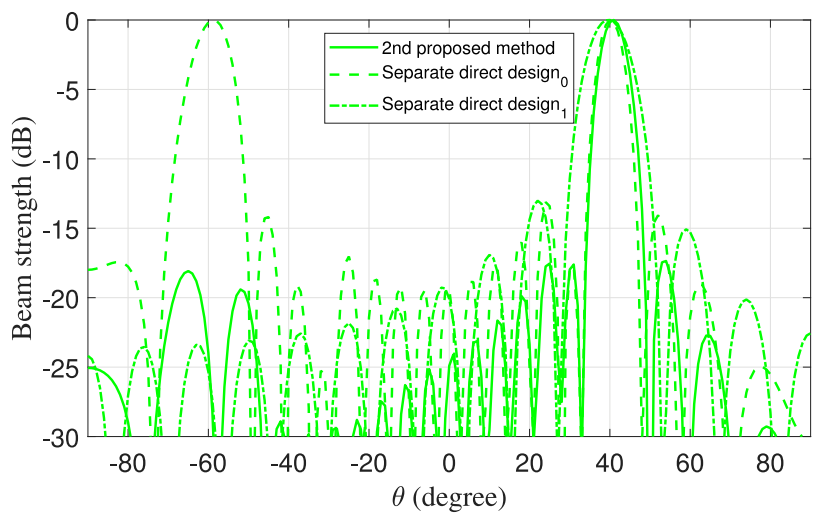

Fig. 13. Beam pattern of the second beam with $\varphi_{2}=40^{\circ}$ for the second proposed scheme in Section IV-Aii) and the separate direct designs with the interleaved and localised architectures using the method in Appendix $(d=$ $\left.\frac{2}{9} \lambda\right)$.
TABLE VI

Digital coefficients $\mathbf{w}_{\mathrm{D}, 0}, \mathbf{w}_{\mathrm{D}, 1}$ and $\mathbf{w}_{\mathrm{D}, 2}$ when $\varphi_{0}=-55^{\circ}$, $\varphi_{1}=-5^{\circ}$ and $\varphi_{2}=40^{\circ}$ for the second proposed scheme in Section IV-Aii) $\left(d=\frac{2}{9} \lambda\right)$.

\begin{tabular}{|c|c|c|c|}
\hline $\mathrm{m}$ & $\mathbf{w}_{\mathrm{D}, 0}$ & $\mathbf{w}_{\mathrm{D}, 1}$ & $\mathbf{w}_{\mathrm{D}, 2}$ \\
\hline 0 & 5.4556 & 0.9683 & 4.7700 \\
\hline 1 & 3.3085 & -0.2793 & 4.0944 \\
\hline 2 & -0.2327 & 0.0070 & 0.4455 \\
\hline
\end{tabular}

TABLE VII

Analogue coefficients $w_{0}, w_{1}$ and $w_{2}$ when $\varphi_{0}=-55^{\circ}, \varphi_{1}=-5^{\circ}$ and $\varphi_{2}=40^{\circ}$ for the second proposed scheme in Section IV-Aii) $\left(d=\frac{2}{9} \lambda\right)$.

\begin{tabular}{|c|c|c|c|}
\hline $\mathrm{n}$ & $\mathbf{w}_{0}$ & $\mathbf{w}_{1}$ & $\mathbf{w}_{2}$ \\
\hline 0 & $0.0165+0.0017 \mathrm{i}$ & $-0.0202-0.0021 \mathrm{i}$ & $0.0755+0.0916 \mathrm{i}$ \\
\hline 1 & $0.0184-0.0057 \mathrm{i}$ & $-0.0411+0.0199 \mathrm{i}$ & $0.0294-0.1041 \mathrm{i}$ \\
\hline 2 & $0.0366-0.0307 \mathrm{i}$ & $-0.0452+0.0496 \mathrm{i}$ & $0.1522-0.0870 \mathrm{i}$ \\
\hline 3 & $0.0203-0.0451 \mathrm{i}$ & $-0.0253+0.0820 \mathrm{i}$ & $-0.0601-0.1700 \mathrm{i}$ \\
\hline 4 & $0.0061-0.0609 \mathrm{i}$ & $0.0014+0.0767 \mathrm{i}$ & $0.0721-0.1488 \mathrm{i}$ \\
\hline 5 & $-0.0163-0.0496 \mathrm{i}$ & $0.0380+0.0820 \mathrm{i}$ & $-0.1750-0.1031 \mathrm{i}$ \\
\hline 6 & $-0.0387-0.0551 \mathrm{i}$ & $0.0586+0.0680 \mathrm{i}$ & $-0.0619-0.2022 \mathrm{i}$ \\
\hline 7 & $-0.0532-0.0345 \mathrm{i}$ & $0.0929+0.0439 \mathrm{i}$ & $-0.2044+0.0554 \mathrm{i}$ \\
\hline 8 & $-0.0635-0.0131 \mathrm{i}$ & $0.0810+0.0044 \mathrm{i}$ & $-0.1623-0.0924 \mathrm{i}$ \\
\hline 9 & $-0.0569+0.0139 \mathrm{i}$ & $0.0917-0.0301 \mathrm{i}$ & $-0.1145+0.1449 \mathrm{i}$ \\
\hline 10 & $-0.0552+0.0243 \mathrm{i}$ & $0.0652-0.0349 \mathrm{i}$ & $-0.1752+0.0275 \mathrm{i}$ \\
\hline 11 & $-0.0317+0.0391 \mathrm{i}$ & $0.0386-0.0732 \mathrm{i}$ & $0.0299+0.1772 \mathrm{i}$ \\
\hline 12 & $-0.0109+0.0500 \mathrm{i}$ & $0.0063-0.0636 \mathrm{i}$ & $-0.0765+0.0943 \mathrm{i}$ \\
\hline 13 & $0.0026+0.0409 \mathrm{i}$ & $-0.0043-0.0551 \mathrm{i}$ & $0.0686+0.0643 \mathrm{i}$ \\
\hline 14 & $-0.0049+0.0154 \mathrm{i}$ & $0.0125-0.0068 \mathrm{i}$ & $-0.0863+0.0597 \mathrm{i}$ \\
\hline
\end{tabular}

Overall, from both sets of design examples, it can be seen that the second proposed method with a fixed antenna spacing is working effectively with the resultant beams pointing to the desired directions, while the separate direct designs based on each subarray using the method in Appendix is not as good and in the interleaved subarray architecture, it even leads to a grating lobe as shown in Fig. 7 for the two-user case and Figs. 11 and 13 for the three-user case due to a spacing larger than half wavelength. Furthermore, based on the performances with two types of sub-array architectures in the above two cases, the interleaved subarray architecture provides a better result with a narrower mainlobe beamwidth than the localised architecture.

Note that when $d=\frac{1}{3} \lambda$ and $\frac{2}{9} \lambda$ for the two and three users, respectively, the antenna spacing for each subarray is $\frac{2}{3} \lambda$ and grating lobes are expected. This also highlights the positive effect of the digital scheme, which can combine the multiple subarrays together in an effective way to eliminate grating lobes.

\section{Design examples for the second proposed scheme based on UPA}

For the design based on UPA with $M=2$, one fixed antenna spacing $d_{x}=d_{y}=\frac{1}{3} \lambda$ is employed for the two users and the number of the antennas in the whole array is $2 N_{x} \times 2 N_{y}=$ 144. In addition, the mainlobe direction in the azimuth angle for both designed beams is selected as $\phi_{m}=0^{\circ}$ and the corresponding sidelobe region is $\phi_{s} \in\left[-90^{\circ},-5^{\circ}\right] \cup\left[5^{\circ}, 90^{\circ}\right]$. The desired elevation angles and the corresponding sidelobe regions for the two designed beams are the same as the twouser case in Section V-C. Moreover, the trade-off factor in the 
weighting function is $\beta=0.65$ and the convergence factor is $\delta=1 \times 10^{-10}$. The two resultant beams by the second proposed scheme are shown in Figs. 14 and 15, where a satisfactory design performance is achieved with both of their sidelobes being lower than $-10 \mathrm{~dB}$.

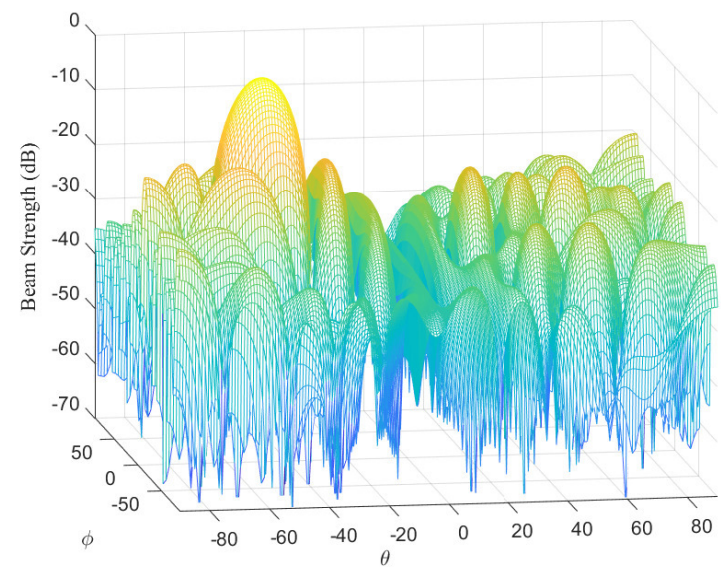

Fig. 14. Beam pattern of the zeroth beam with $\varphi_{0}=-48^{\circ}$ and $\phi_{m}=0^{\circ}$ for the second proposed scheme in Section IV-B $\left(d_{x}=d_{y}=\frac{1}{3} \lambda\right)$.

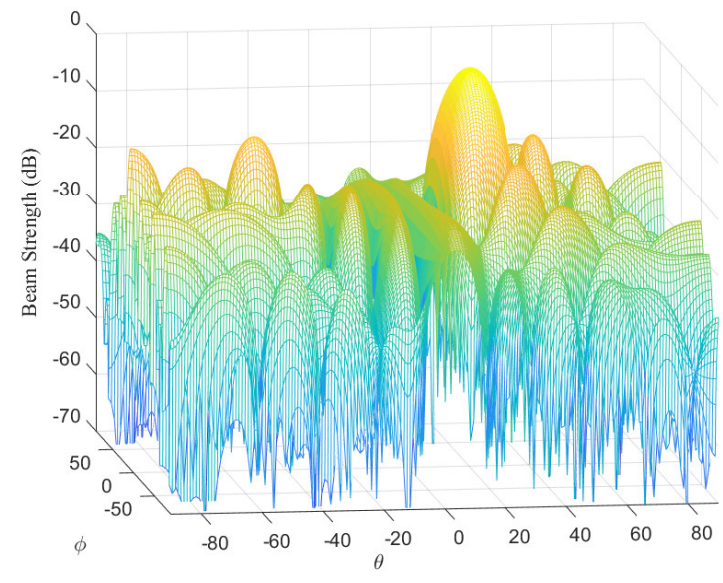

Fig. 15. Beam pattern of the first beam with $\varphi_{1}=20^{\circ}$ and $\phi_{m}=0^{\circ}$ for the second proposed scheme in Section IV-B $\left(d_{x}=d_{y}=\frac{1}{3} \lambda\right)$.

\section{CONCLUSION}

In this paper, two millimetre-wave beam multiplexing design schemes, with varying and fixed antenna spacings, respectively, have been proposed based on the interleaved subarray architecture. The two new designs overcome the limitation of an existing one so that with one set of analogue coefficients, multiple beams serving multiple arbitrary user directions can be generated. As demonstrated by provided design examples based on both ULAs and UPAs, the first proposed design works effectively but the antenna spacing is not fixed any more, while using the least squares approach, the second design achieves multi-beam multiplexing for arbitrary directions by considering the antenna spacing as a fixed parameter again.

\section{APPENDIX}

To show the improvement by the proposed hybrid beamforming design methods from another angle, we consider a least squares design based on three other structures: design based on the interleaved subarray only without any intersubarray coding scheme, design based on the localised subarray only without any inter-subarray coding scheme, and design based on the whole original array. That is, for the first two cases, the $m$-th subarray generates a beam pointing to $\varphi_{x}$ with $m=x$, and all subarrays operate independent of each other; For the third case, the whole array is used directly to form a beam (without inter-subarray coding and without the hybrid structure, just a classic beamformer with the same number of coefficients as the number of antennas).

The starting point is to obtain the steering vector of the considered array first. For the first case based on a ULA, the steering vector has been given by (21). For the second case based on a ULA, i.e., the localised case, its steering vector is given by

$$
\begin{aligned}
\mathbf{S}_{\mathrm{m}}(\theta)= & {\left[e^{j 2 \pi m N_{s} \frac{d}{\lambda} \sin \theta}, e^{j 2 \pi\left(m N_{s}+1\right) \frac{d}{\lambda} \sin \theta},\right.} \\
& \left.\ldots, e^{j 2 \pi\left((m+1) N_{s}-1\right) \frac{d}{\lambda} \sin \theta}\right]^{\mathrm{T}} .
\end{aligned}
$$

For the second case based on a UPA, as shown in Fig. 3, the steering vectors of the two localised planar subarrays are given by

$$
\begin{aligned}
& \mathbf{s}_{0}(\theta, \phi)= \\
& {\left[1, e^{j 2 \pi \frac{d_{y}}{\lambda} \sin \theta \sin \phi}, \ldots, e^{j 2 \pi \frac{\left(N_{y}-1\right) d_{y}}{\lambda} \sin \theta \sin \phi},\right.} \\
& \ldots, e^{j 2 \pi \frac{\left(N_{x}-1\right) d_{x}}{\lambda} \sin \theta \cos \phi}, \ldots, \\
& e^{j 2 \pi\left(\frac{\left(N_{x}-1\right) d_{x}}{\lambda} \sin \theta \cos \phi+\frac{\left(N_{y}-1\right) d_{y}}{\lambda} \sin \theta \sin \phi\right)}, \\
& e^{j 2 \pi\left(\frac{N_{x} d_{x}}{\lambda} \sin \theta \cos \phi+\frac{N_{y} d_{y}}{\lambda} \sin \theta \sin \phi\right)}, \\
& \left.\ldots, e^{j 2 \pi\left(\frac{\left(2 N_{x}-1\right) d_{x}}{\lambda} \sin \theta \cos \phi+\frac{\left(2 N_{y}-1\right) d_{y}}{\lambda} \sin \theta \sin \phi\right)}\right]^{\mathrm{T}}, \\
& \mathbf{s}_{1}(\theta, \phi)= \\
& {\left[e^{j 2 \pi \frac{N_{y} d_{y}}{\lambda} \sin \theta \sin \phi}, \ldots, e^{j 2 \pi \frac{\left(2 N_{y}-1\right) d_{y}}{\lambda} \sin \theta \sin \phi}, \ldots,\right.} \\
& e^{j 2 \pi\left(\frac{\left(N_{x}-1\right) d_{x}}{\lambda} \sin \theta \cos \phi+\frac{N_{y} d_{y}}{\lambda} \sin \theta \sin \phi\right)}, \ldots, \\
& e^{j 2 \pi\left(\frac{\left(N_{x}-1\right) d_{x}}{\lambda} \sin \theta \cos \phi+\frac{\left(2 N_{y}-1\right) d_{y}}{\lambda} \sin \theta \sin \phi\right)}, \\
& e^{j 2 \pi \frac{N_{x} d_{x}}{\lambda} \sin \theta \cos \phi}, \ldots, e^{j 2 \pi\left(\frac{N_{x} d_{x}}{\lambda} \sin \theta \cos \phi+\frac{\left(N_{y}-1\right) d_{y}}{\lambda} \sin \theta \sin \phi\right)}, \\
& \left.\ldots, e^{j 2 \pi\left(\frac{\left(2 N_{x}-1\right) d_{x}}{\lambda} \sin \theta \cos \phi+\frac{\left(N_{y}-1\right) d_{y}}{\lambda} \sin \theta \sin \phi\right)}\right]^{\mathrm{T}} .
\end{aligned}
$$

For the third case, the steering vector based on a ULA is given by

$$
\mathbf{s}(\theta)=\left[1, e^{j 2 \pi \frac{d}{\lambda} \sin \theta}, e^{j 2 \pi 2 \frac{d}{\lambda} \sin \theta}, \ldots, e^{j 2 \pi\left(2 N_{s}-1\right) \frac{d}{\lambda} \sin \theta}\right]^{\mathrm{T}} .
$$

Now we use $\mathbf{s}(\theta)$ to represent a general steering vetor and $\mathbf{w}$ to represent the corresponding beamforming coefficients. Then the cost formulation of the beam pointing to $\varphi_{x}$ generated by the array is given by

$$
\begin{aligned}
J_{\mathrm{LS}} & =(1-\beta) \sum_{\theta \in \Theta_{\mathrm{m}_{\mathbf{x}}}}\left|\mathbf{w}^{\mathrm{H}} \mathbf{s}(\theta)-1\right|^{2} \\
& +\left(\beta / N_{g}\right) \sum_{\theta \in \Theta_{\mathrm{s}_{\mathbf{x}}}}\left|\mathbf{w}^{\mathrm{H}} \mathbf{s}(\theta)\right|^{2},
\end{aligned}
$$


where $\Theta_{m_{x}}$ represents the main lobe area and $\Theta_{\mathrm{s}_{\mathrm{x}}}$ represents the sidelobe region.

Equation (83) can be expanded into a quadratic form

$$
J_{\mathrm{LS}}=\mathbf{w}^{\mathrm{H}} \mathbf{Q}_{\mathrm{LS}} \mathbf{x q}-\mathbf{w}^{\mathrm{H}} \mathbf{z}_{\mathrm{LS}_{\mathrm{xq}}}-\mathbf{z}_{\mathrm{LS}}^{\mathrm{H}} \mathbf{x q}+d_{\mathrm{LS}_{\mathbf{x}}},
$$

where $\mathbf{Q}_{\mathrm{LS}_{\mathrm{xq}}}, \mathbf{z}_{\mathrm{LS}}$, and $d_{\mathrm{LS}_{\mathrm{x}}}$ have been defined by (30), (33), and (34) using corresponding types of steering vectors. Overall, the minimisation of $(84)$ with respect to $\mathrm{w}^{\mathrm{H}}$, gives the following solutions

$$
\mathbf{w}=\mathbf{Q}_{\mathrm{LS}_{\mathrm{xq}}}^{-1} \mathbf{z}_{\mathrm{LS}} .
$$

\section{REFERENCES}

[1] F. Boccardi, R. W. Heath, A. Lozano, T. L. Marzetta, and P. Popovski, "Five disruptive technology directions for 5G," IEEE Commun. Mag., vol. 52, no. 2, pp. 74-80, Feb. 2014.

[2] S. A. Busari, K. M. S. Huq, S. Mumtaz, et al., "Generalized hybrid beamforming for vehicular connectivity using THz massive MIMO," IEEE Trans. Veh. Technol., vol. 68, no. 9, pp. 8372-8383, Sept. 2019.

[3] K. Satyanarayana, M. El-Hajjar, P. Kuo, A. Mourad, and L. Hanzo, "Hybrid beamforming design for full-duplex millimeter wave communication," IEEE Trans. Veh. Technol., vol. 68, no. 2, pp. 1394-1404, Feb. 2019.

[4] S. Payami, M. Ghoraishi, M. Dianati, and M. Sellathurai, "Hybrid beamforming with a reduced number of phase shifters for massive MIMO systems," IEEE Trans. Veh. Technol., vol. 67, no. 6, pp. 48434851, June 2018.

[5] Z. Zheng and H. Gharavi, "Spectral and energy efficiencies of millimeter wave MIMO with configurable hybrid precoding," IEEE Trans. Veh. Technol., vol. 68, no. 6, pp. 5732-5746, June 2019.

[6] M. M. Molu, P. Xiao, M. Khalily, K. Cumanan, L. Zhang, and R. Tafazolli, "Low-complexity and robust hybrid beamforming design for multiantenna communication systems," IEEE Trans. Wireless Commun., vol 17, no. 3, pp. 1445-1459, Mar. 2018.

[7] A. Li and C. Masouros, "Energy-efficient SWIPT: From fully digital to hybrid analog-digital beamforming," IEEE Trans. Veh. Technol., vol. 67, no. 4, pp. 3390-3405, Apr. 2018.

[8] Q. Luo, S. Gao, W. Liu, and C. Gu, Low-cost Smart Antennas, Wiley Press, Mar. 2019.

[9] W. Roh, J. Seol, J. Park, et al., "Millimeter-wave beamforming as an enabling technology for 5G cellular communications: Theoretical feasibility and prototype results," IEEE Commun. Mag., vol. 53, no. 2, pp. 106-113, Feb. 2014.

[10] S. Han, C. I, Z. Xu, and C. Rowell, "Large-scale antenna systems with hybrid analog and digital beamforming for millimeter wave 5G," IEEE Commun. Mag., vol. 53, no. 1, pp. 186-194, Jan. 2015.

[11] A. F. Molisch, V. V. Ratnam, S. Han, et al., "Hybrid beamforming for massive MIMO: A survey," IEEE Commun. Mag., vol. 55, no. 9, pp. 134-141, Sept. 2017.

[12] F. Sohrabi and W. Yu, "Hybrid analog and digital beamforming for mmWave OFDM large-scale antenna arrays," IEEE J. Sel. Areas Commun., vol. 35, no. 7, pp. 1432-1443, July 2017.

[13] V. Venkateswaran and A. van der Veen, "Analog beamforming in MIMO communications with phase shift networks and online channel estimation," IEEE Trans. Signal Process., vol. 58, no. 8, pp. 4131-4143, Aug. 2010.

[14] O. Oliaei, "A two-antenna low-IF beamforming MIMO receiver," in Proc. IEEE Global Communications Conference, Washington, DC, USA, Nov. 2007, pp. 3591-3595.

[15] C. Miller, W. Liu, and R. J. Langley, "Reduced complexity MIMO receiver with real-valued beamforming," in Proc. IEEE International Conference on Computer and Information Technology, Liverpool, UK, Oct. 2015, pp. 31-36.

[16] W. Liu and S. Weiss, Wideband Beamforming: Concepts and Techniques, John Wiley \& Sons, Mar. 2010.

[17] X. Huang and Y. Guo, "Frequency-domain AoA estimation and beamforming with wideband hybrid arrays," IEEE Trans. Wireless Commun., vol. 10, no. 8, pp. 2543-2553, Aug. 2011.

[18] T. S. Rappaport, S. Sun, R. Mayzus, et al., "Millimeter wave mobile communications for 5G cellular: It will work!," IEEE Access, vol. 1, pp. 335-349, May 2013.
[19] J. A. Zhang, X. Huang, V. Dyadyuk, and Y. J. Guo, "Massive hybrid antenna array for millimeter-wave cellular communications," IEEE Wirel. Commun., vol. 22, no. 1, pp. 79-87, Feb. 2015.

[20] Ching-Tai Lin and Hung Ly, "Sidelobe reduction through subarray overlapping for wideband arrays," in Proc. IEEE Radar Conference, Atlanta, GA, USA, May 2001, pp. 228-233.

[21] Y. Guo, X. Huang, and V. Dyadyuk, "A hybrid adaptive antenna array for long-range mm-wave communications," IEEE Antennas Propag. Mag., vol. 54, no. 2, pp. 271-282, Apr. 2012.

[22] S. Fujio, C. Kojima, T. Shimura, et al., "Robust beamforming method for SDMA with interleaved subarray hybrid beamforming," in Proc. IEEE 27th Annual International Symposium on Personal, Indoor, and Mobile Radio Communications (PIMRC), Valencia, Spain, Sept. 2016, pp. 1-5.

[23] T. Shimura, T. Ohshima, H. Ashida, et al., "Millimeter-wave TX phased array with phase adjusting function between transmitters for hybrid beamforming with interleaved subarrays," in Proc. 46th European Microwave Conference (EuMC), London, UK, Oct. 2016, pp. 15721575.

[24] P. Rocca, R. Haupt, and A. Massa, "Sidelobe reduction through element phase control in uniform subarrayed array antennas," IEEE Antennas Wireless Propag. Lett., vol. 8, no. 1, pp. 437-440, Feb. 2009.

[25] Z. Li, A. Honda, T. Shimura, et al., "Multi-user mmwave communication by interleaved beamforming with inter-subarray coding," in Proc. IEEE 28th Annual International Symposium on Personal, Indoor, and Mobile Radio Communications (PIMRC), Montreal, QC, Canada, Oct. 2017, pp. $1-6$.

[26] M. Shimizu, "Millimeter-wave beam multiplexing method using subarray type hybrid beamforming of interleaved configuration with intersubarray coding," Int. J. Wirel. Inf. Netw., vol. 24, no. 3, pp. 217-224, Sept. 2017.

[27] J. Zhang, W. Liu, C. Gu, S. Gao, and Q. Luo, "Two-beam multiplexing with inter-subarray coding for arbitrary directions based on interleaved subarray architectures," in Proc. IEEE 30th Annual International Symposium on Personal, Indoor and Mobile Radio Communications (PIMRC), Istanbul, Turkey, Sept. 2019, pp. 1-5.

[28] M. Shimizu, A. Honda, S. Ishikawa, et al., "Millimeter-wave beam multiplexing method using hybrid beamforming," in Proc. IEEE 27th Annual International Symposium on Personal, Indoor, and Mobile Radio Communications (PIMRC), Valencia, Spain, Sept. 2016, pp. 1-6.

[29] S. Doclo and M. Moonen, "Design of broadband beamformers robust against gain and phase errors in the microphone array characteristics," IEEE Trans. Signal Process., vol. 51, no. 10, pp. 2511-2526, Oct. 2003.

[30] Y. Zhao, W. Liu, and R. Langley, "Application of the least squares approach to fixed beamformer design with frequency-invariant constraints," IET Signal Processing, vol. 5, no. 3, pp. 281-291, June 2011.

[31] S. Kim and Y. E. Wang, "Two-dimensional planar array for digital beamforming and direction-of-arrival estimations," IEEE Trans. Veh. Technol., vol. 58, no. 7, pp. 3137-3144, Sept. 2009.

[32] W. Liu, "Design and implementation of a rectangular frequency invariant beamformer with a full azimuth angle coverage," J Franklin Inst, vol. 348 , no. 9, pp. 2556 - 2569, Nov. 2011.

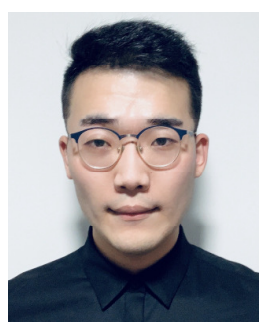

Junwei Zhang received the B.Eng. degree from the Dalian Maritime University, China, in 2016, and the M.Sc. degree from the Department of Electronic and Electrical Engineering, The University of Sheffield, in 2017 , where he is currently pursuing the $\mathrm{Ph}$. D. degree. His research interests include array signal processing (beamforming based on interleaved subarray architecture) and multi-beam multiplexing. 


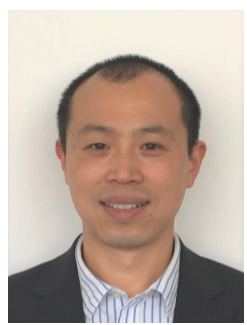

Wei Liu (S'01-M'04-SM'10) received his BSc and LLB. degrees from Peking University, China, in 1996 and 1997, respectively, MPhil from the University of Hong Kong in 2001, and PhD from the School of Electronics and Computer Science, University of Southampton, UK, in 2003. He then worked as a postdoc first at Southampton and later at the Department of Electrical and Electronic Engineering, Imperial College London. Since September 2005, he has been with the Department of Electronic and Electrical Engineering, University of Sheffield, UK first as a Lecturer and then a Senior Lecturer. He has published more than 300 journal and conference papers, five book chapters, and two research monographs titled "Wideband Beamforming: Concepts and Techniques" (John Wiley, March 2010) and "Low-Cost Smart Antennas" (by Wiley-IEEE, March 2019), respectively. His research interests cover a wide range of topics in signal processing, with a focus on sensor array signal processing and its various applications, such as robotics and autonomous systems, human computer interface, radar, sonar, satellite navigation, and wireless communications

$\mathrm{He}$ is a member of the Digital Signal Processing Technical Committee of the IEEE Circuits and Systems Society and the Sensor Array and Multichannel Signal Processing Technical Committee of the IEEE Signal Processing Society (Vice-Chair from Jan 2019). He was an Associate Editor for IEEE Trans. on Signal Processing (March 2015-March 2019) and is currently an Associate Editor for IEEE Access, and an editorial board member of the journal Frontiers of Information Technology and Electronic Engineering.

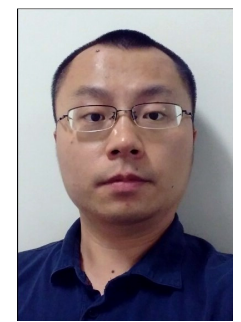

Qi Luo (S'08-M'12) was born in Chengdu, China in 1982. He received his MSc degree with distinction from the University of Sheffield, the UK in 2006 and his $\mathrm{PhD}$ degree from University of Porto, Portugal with distinction in 2012. From 2012-2013, he worked at Surrey space centre, UK as a research fellow. Currently, he is working at the School of Engineering and Digital Arts, University of Kent, the UK as a research fellow. His research interests are smart antennas, circularly polarized antennas, reflectarray, transmitarray, multiband microstrip antennas, and electrically small antenna design. He authored/co-authored two books Circularly Polarized Antennas (Wiley-IEEE, 2014) and Low-Cost Smart Antennas (Wiley, 2019). He also authored a book chapter in Handbook of Antenna Technologies (Springer Singapore, 2014). He has been serving as a reviewer for several technical journals and international conferences and was awarded as the Outstanding reviewers for IEEE Transactions on Antennas and Propagation in 2015

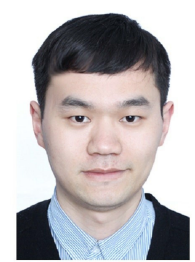

Chao Gu received the B.S. and M.S. degrees from Xidian University, Xi' an, China, in 2009 and 2012, respectively, and the Ph.D. degree from the University of Kent, Canterbury, U.K., in 2017. He is currently with the Centre for Wireless Innovation, ECIT Institute, School of Electronics, Electrical Engineering and Computer Science, Queen's University Belfast, Belfast, U.K. His research interests include phased array antennas, reconfigurable antennas, and frequency selective surfaces.

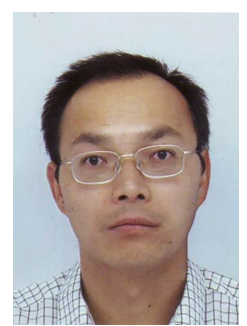

Steven Shichang Gao (M'01-SM'16-F'19) received the $\mathrm{PhD}$ from Shanghai University, China. He is a Full Professor and Chair in RF and Microwave Engineering, and the Director of Graduate Studies at the School of Engineering and Digital Arts, University of Kent, UK. His research covers smart antennas, phased arrays, MIMO, reconfigurable antennas, broadband/multiband antennas, satellite antennas, $\mathrm{RF} /$ microwave/mm-wave/THz circuits, mobile communications, satellite communications, UWB radars, synthetic-aperture radars, IOT and sensors for healthcare. He co-authored/co-edited three books (Space Antenna Handbook, Wiley, 2012; Circularly Polarized Antennas, IEEE-Wiley, 2014; Low-Cost Smart Antennas, Wiley, 2019), over 400 papers and 10 patents. He was a Distinguished Lecturer of IEEE Antennas and Propagation Society, and serves as an Associate Editor for several international Journals (IEEE Transactions on Antennas and Propagation; Radio Science; Electronics Letters; IET Circuits, Devices and Systems, etc), and the Editor-in-Chief for John Wiley \& Sons Book Series on "Microwave and Wireless Technologies". He was General Chair of LAPC 2013, and an Invited Speaker at many conferences. He is a Fellow of IEEE, and also a Fellow of Royal Aeronautical Society, UK, and the IET, UK. 